\title{
On Naturally Graded Lie and Leibniz Superalgebras
}

\author{
L. M. Camacho ${ }^{1}$ • R. M. Navarro ${ }^{2}$ J. M. Sánchez ${ }^{3}$
}

\begin{abstract}
In general, the study of gradations has always represented a cornerstone in the study of non-associative algebras. In particular, natural gradation can be considered to be the first and most relevant gradation of nilpotent Leibniz (resp. Lie) algebras. In fact, many families of relevant solvable Leibniz (resp. Lie) algebras have been obtained by extensions of naturally graded algebras, i.e., solvable algebras with a wellstructured nilradical. Thus, the aim of this work is introducing the concept of natural gradation for Lie and Leibniz superalgebras. Moreover, after having defined naturally graded Lie and Leibniz superalgebras, we characterize natural gradations on a very important class of each of them, that is, those with maximal supernilindex.
\end{abstract}

Keywords Lie (super)algebras · Cohomology $\cdot$ Deformation $\cdot$ Leibniz (super)algebras $\cdot$ Naturally graded

Mathematics Subject Classification 17A32 $\cdot 17 \mathrm{~B} 30 \cdot 17 \mathrm{~B} 70 \cdot 17 \mathrm{~A} 70$

This work has been supported by Agencia Estatal de Investigación (Spain), Grant MTM2016-79661-P (European FEDER support included, UE), by the PCI of the UCA "Teoría de Lie y Teoría de Espacios de Banach" and by the PAI with project number FQM298.

$凶$ L. M. Camacho

lcamacho@us.es

R. M. Navarro

rnavarro@unex.es

J. M. Sánchez

txema.sanchez@uca.es

1 Dpto. Matemática Aplicada I, Universidad de Sevilla, Sevilla, Spain

2 Dpto. de Matemáticas, Universidad de Extremadura, Cáceres, Spain

3 Dpto. de Matemáticas, Universidad de Cádiz, Campus de Puerto Real, Cádiz, Spain 


\section{Introduction}

In general, the study of graded algebras has always played a fundamental role into Lie theory (see for instance $[10,15]$ ). Recently, the importance of naturally graded Lie and Leibniz algebras has been increased by means of the use of them as nilradical of relevant families of solvable ones (see $[1,5,13,28]$ ). Therefore, it remains as an immediate and future work and the use of the results of the present paper to obtain important families of solvable Lie and Leibniz superalgebras by extensions of nonnilpotent outer derivations. Recall that filiform Lie algebras were firstly introduced in [34] and the generalization for Lie superalgebras has already been obtained (see [8]) and, in the same way as occurs for Lie algebras, filiform Lie superalgebras have maximal supernilindex. On the other hand, the notion of Leibniz superalgebras as a generalization of Leibniz algebras was firstly introduced in [2], and general graded Leibniz algebras were considered before in work [29], though. Since Leibniz algebras are a generalization of Lie algebras [30], consequently many of the features of Leibniz superalgebras are generalization of Lie superalgebras $[3,11,12,19]$. Likewise, the study of nilpotent Leibniz algebras $[2,6,7]$ can be very useful to study nilpotent Leibniz superalgebras.

Thus, we begin the present work introducing the concept of naturally graded for Lie and Leibniz superalgebras. Secondly we tackle the problem of determining which filiform Lie superalgebras are naturally graded obtaining a complete classification (up to isomorphism) for total dimension less or equal to 7 as well as some other classifications. Finally, we characterize naturally graded Leibniz superalgebras with maximal supernilindex. Note that the techniques used for Leibniz superalgebras are totally different from the ones used for Lie superalgebras and involve a huge amount of computation.

Throughout the present paper we will consider vector spaces and algebras over the field of complex numbers $\mathbb{C}$.

\section{Preliminary Results}

\subsection{Preliminary Results for Lie Superalgebras}

A vector space $V$ is said to be $\mathbb{Z}_{2}$-graded if it admits a decomposition in direct sum, $V=V_{\overline{0}} \oplus V_{\overline{1}}$, where $\overline{0}, \overline{1} \in \mathbb{Z}_{2}$. An element $X \in V$ is called homogeneous of degree $\bar{i}$ if it is an element of $V_{\bar{i}}, \bar{i} \in \mathbb{Z}_{2}$. In particular, the elements of $V_{\overline{0}}$ (resp. $V_{\overline{1}}$ ) are also called even (resp. odd). A Lie superalgebra (see [14,32]) is a $\mathbb{Z}_{2}$-graded vector space $\mathfrak{g}=\mathfrak{g}_{\overline{0}} \oplus \mathfrak{g}_{\overline{1}}$, with an even bilinear commutation operation (or "supercommutation") $[\cdot, \cdot]$, which satisfies the conditions

1. $[X, Y]=-(-1)^{\bar{i} \bar{j}}[Y, X]$,

2. $(-1)^{\bar{k} \bar{k}}[X,[Y, Z]]+(-1)^{\bar{i} \bar{j}}[Y,[Z, X]]+(-1)^{\bar{j} \bar{k}}[Z,[X, Y]]=0$ (super Jacobi identity)

for all $X \in \mathfrak{g}_{\bar{i}}, Y \in \mathfrak{g}_{\bar{j}}, Z \in \mathfrak{g}_{\bar{k}}$ with $\bar{i}, \bar{j}, \bar{k} \in \mathbb{Z}_{2}$. 
Thus, $\mathfrak{g}_{\overline{0}}$ is an ordinary Lie algebra, and $\mathfrak{g}_{\overline{1}}$ is a module over $\mathfrak{g}_{\overline{0}}$; the Lie superalgebra structure also contains the symmetric pairing $S^{2} \mathfrak{g}_{\overline{1}} \longrightarrow \mathfrak{g}_{\overline{0}}$. Hereafter, any time we write $\left[X_{i}, X_{j}\right]$ or $\left[X_{i}, Y_{j}\right]$ with $X_{i}$ even and $Y_{j}$ odd elements, and the products $\left[X_{j}, X_{i}\right],\left[Y_{j}, X_{i}\right]$ are assumed to be obtained by the skew-symmetric property as it is usual in Lie theory. Also, for simplicity the symmetric products $\left[Y_{i}, Y_{j}\right]=\left[Y_{j}, Y_{i}\right]$ are denoted by $\left(Y_{i}, Y_{j}\right)$. Note that when we deal with Leibniz superalgebras we do not have skew-symmetric nor symmetric property and each single bracket product must be specified.

In general, the descending central sequence of a Lie superalgebra $\mathfrak{g}=\mathfrak{g}_{\overline{0}} \oplus \mathfrak{g}_{\overline{1}}$ is defined in the same way as for Lie algebras: $\mathcal{C}^{0}(\mathfrak{g}):=\mathfrak{g}, \mathcal{C}^{k+1}(\mathfrak{g}):=\left[\mathcal{C}^{k}(\mathfrak{g}), \mathfrak{g}\right]$ for all $k \geq 0$. Consequently, if $\mathcal{C}^{k}(\mathfrak{g})=\{0\}$ for some $k$, then the Lie superalgebra is called nilpotent. Nevertheless, there are also defined two other descending sequences called $\mathcal{C}^{k}\left(\mathfrak{g}_{0}\right)$ and $\mathcal{C}^{k}\left(\mathfrak{g}_{1}\right)$ which will be important in our study. They are defined by $\mathcal{C}^{0}\left(\mathfrak{g}_{\bar{i}}\right):=\mathfrak{g}_{\bar{i}}, \mathcal{C}^{k+1}\left(\mathfrak{g}_{\bar{i}}^{-}\right):=\left[\mathfrak{g}_{\overline{0}}, \mathcal{C}^{k}\left(\mathfrak{g}_{\bar{i}}^{-}\right)\right], \quad k \geq 0, \bar{i} \in \mathbb{Z}_{2}$.

If $\mathfrak{g}=\mathfrak{g}_{0} \oplus \mathfrak{g}_{\overline{1}}$ is a nilpotent Lie superalgebra, then $\mathfrak{g}$ has supernilindex or $s$-nilindex $(p, q)$ if satisfies

$$
\left(\mathcal{C}^{p-1}\left(\mathfrak{g}_{0}\right)\right) \neq 0, \quad\left(\mathcal{C}^{q-1}\left(\mathfrak{g}_{\overline{1}}\right)\right) \neq 0, \quad \mathcal{C}^{p}\left(\mathfrak{g}_{\overline{0}}\right)=\mathcal{C}^{q}\left(\mathfrak{g}_{\overline{1}}\right)=0
$$

Note that a module $A=A_{\overline{0}} \oplus A_{\overline{1}}$ of the Lie superalgebra $\mathfrak{g}$ is an even bilinear map $\mathfrak{g} \times A \rightarrow A$ satisfying

$$
X(Y a)-(-1)^{\bar{i} \bar{j}} Y(X a)=[X, Y] a
$$

for all $X \in \mathfrak{g}_{i}, Y \in \mathfrak{g}_{\bar{j}}, \bar{i}, \bar{j} \in \mathbb{Z}_{2}$, and $a \in A$.

Lie superalgebra cohomology is defined in the following well-known way ( see, e.g., $[14,33]$ ): the $\mathbb{Z}_{2}$-graded vector space of $q$-dimensional cocycles of the Lie superalgebra $\mathfrak{g}=\mathfrak{g}_{\overline{0}} \oplus \mathfrak{g}_{\overline{1}}$ with coefficients in the $\mathfrak{g}$-module $A=A_{\overline{0}} \oplus A_{\overline{1}}$ is given by

$$
C^{q}(\mathfrak{g} ; A):=\bigoplus_{q_{0}+q_{1}=q} \operatorname{Hom}\left(\wedge^{q_{0}} \mathfrak{g}_{\overline{0}} \otimes S^{q_{1}} \mathfrak{g}_{\overline{1}}, A\right)
$$

The above space is $\mathbb{Z}_{2}$-graded as $C^{q}(\mathfrak{g} ; A)=C_{0}^{q}(\mathfrak{g} ; A) \oplus C_{1}^{q}(\mathfrak{g} ; A)$ being

$$
C_{p}^{q}(\mathfrak{g} ; A):=\bigoplus_{\substack{q_{0}+q_{1}=q \\ q_{1}+r \equiv p \bmod 2}} \operatorname{Hom}\left(\wedge^{q_{0}} \mathfrak{g}_{\overline{0}} \otimes S^{q_{1}} \mathfrak{g}_{\overline{1}}, A_{r}\right)
$$

Thus we have the cohomology groups

$$
H_{p}^{q}(\mathfrak{g} ; A):=Z_{p}^{q}(\mathfrak{g} ; A) / B_{p}^{q}(\mathfrak{g} ; A)
$$

where, in particular, the elements of $Z_{0}^{q}(\mathfrak{g} ; A)$ and $Z_{1}^{q}(\mathfrak{g} ; A)$ are called even $q$-cocycles and odd q-cocycles, respectively. We denote the variety of nilpotent Lie superalgebras $\mathfrak{g}=\mathfrak{g}_{\overline{0}} \oplus \mathfrak{g}_{\overline{1}}$ with $\operatorname{dim}\left(\mathfrak{g}_{0}\right)=n+1$ and $\operatorname{dim}\left(\mathfrak{g}_{\overline{1}}\right)=m$ with the notation $\mathcal{N}^{n+1, m}$, in 
complete analogy to Lie algebras (see [23,24]). Then, any nilpotent Lie superalgebra $\mathfrak{g}=\mathfrak{g}_{\overline{0}} \oplus \mathfrak{g}_{\overline{1}} \in \mathcal{N}^{n+1, m}$ with s-nilindex $(n, m)$ is called filiform [16].

We denote by $\mathcal{F}^{n+1, m}$ the subset of $\mathcal{N}^{n+1, m}$ consisting of all the filiform Lie superalgebras. Likewise, it can be seen that any filiform Lie superalgebra can be always expressed in a suitable basis or so-called adapted basis. Therefore, if $\mathfrak{g}=\mathfrak{g}_{\overline{0}} \oplus \mathfrak{g}_{\overline{1}} \in$ $\mathcal{F}^{n+1, m}$, then there exists an adapted basis of $\mathfrak{g}$, namely $\left\{X_{0}, X_{1}, \ldots, X_{n}, Y_{1}, \ldots, Y_{m}\right\}$, with $\left\{X_{0}, X_{1}, \ldots, X_{n}\right\}$ a basis of $\mathfrak{g}_{0}$ and $\left\{Y_{1}, \ldots, Y_{m}\right\}$ a basis of $\mathfrak{g}_{\overline{1}}$, such that

$$
\begin{aligned}
& {\left[X_{0}, X_{i}\right]=X_{i+1}, 1 \leq i \leq n-1, \quad\left[X_{0}, X_{n}\right]=0} \\
& {\left[X_{0}, Y_{j}\right]=Y_{j+1}, 1 \leq j \leq m-1,\left[X_{0}, Y_{m}\right]=0}
\end{aligned}
$$

From this result it can be observed that the simplest filiform Lie superalgebra or so-called the model filiform Lie superalgebra, denoted by $L^{n, m}$, will be defined by the only nonzero bracket products

$$
L^{n, m}:\left\{\begin{array}{l}
{\left[X_{0}, X_{i}\right]=X_{i+1}, 1 \leq i \leq n-1} \\
{\left[X_{0}, Y_{j}\right]=Y_{j+1}, 1 \leq j \leq m-1}
\end{array}\right.
$$

$L^{n, m}$ will be the most important filiform Lie superalgebra, in complete analogy to Lie algebras, since all the other filiform Lie superalgebras can be obtained from it by deformations. These infinitesimal deformations will be given by the even 2-cocycles, $Z_{0}^{2}\left(L^{n, m}, L^{n, m}\right)$, which can be decomposed in the way

$$
\begin{aligned}
Z_{0}^{2}\left(L^{n, m}, L^{n, m}\right)= & \underbrace{Z^{2}\left(L^{n, m}, L^{n, m}\right) \cap \operatorname{Hom}\left(\mathfrak{g}_{\overline{0}} \wedge \mathfrak{g}_{\overline{0}}, \mathfrak{g}_{\overline{0}}\right)}_{C} \oplus \underbrace{\underbrace{2}\left(L^{n, m}, L^{n, m}\right) \cap \operatorname{Hom}\left(\mathfrak{g}_{\overline{0}} \wedge \mathfrak{g}_{\overline{1}}, \mathfrak{g}_{\overline{1}}\right)}_{A} \oplus \\
\underbrace{Z^{2}\left(L^{n, m}, L^{n, m}\right) \cap \operatorname{Hom}\left(S^{2} \mathfrak{g}_{\overline{1}}, \mathfrak{g}_{\overline{0}}\right)}_{B} & =A \oplus B \oplus C
\end{aligned}
$$

where $\mathfrak{g}_{\overline{0}}=L_{\overline{0}}^{n, m}$ and $\mathfrak{g}_{\overline{1}}=L_{\overline{1}}^{n, m}$. The component $C$ has been determined in [8,17], and components $A, B$ have been determined in [26] and [25], respectively. Moreover, using these deformations it has been obtained a complete classification (up to isomorphism) of complex filiform Lie superalgebras of dimension less or equal to 7 (for more details see [31]). The main result used for this classification is the fact that every filiform Lie superalgebra can be obtained by $L^{n, m}+\Psi$ with $\Psi$ an infinitesimal deformation verifying $\Psi \circ \Psi(x, y, z)=\Psi(\Psi(x, y), z)+\Psi(\Psi(z, x), y)+\Psi(\Psi(y, z), x)=0$.

\subsection{Preliminary Results for Leibniz Superalgebras}

Many results and definitions of the above section can be extended for Leibniz superalgebras.

Definition 2.1 [2]. A $\mathbb{Z}_{2}$-graded vector space $L=L_{\overline{0}} \oplus L_{\overline{1}}$ is called a Leibniz superalgebra if it is equipped with a product $[\cdot, \cdot]$ which satisfies the condition

$$
[x,[y, z]]=[[x, y], z]-(-1)^{\bar{i} \bar{j}}[[x, z], y] \text { (super Leibniz identity) }
$$


for all $x \in L, y \in L_{\bar{i}}, z \in L_{\bar{j}}, \bar{i}, \bar{j} \in \mathbb{Z}_{2}$.

Note that if a Leibniz superalgebra $L$ satisfies the identity $[x, y]=-(-1)^{\bar{i} \bar{j}}[y, x]$ for $x \in L_{\bar{i}}, y \in L_{\bar{j}}$, then the super Leibniz identity becomes the super Jacobi identity. Therefore Leibniz superalgebras are a generalization of Lie superalgebras. In the same way as for Lie superalgebras, isomorphisms are assumed to be consistent with the $\mathbb{Z}_{2}$ graduation.

If we denote by $R_{x}$ the right multiplication operator, i.e., $R_{x}: L \rightarrow L$ given as $R_{x}(y):=[y, x]$ for $y \in L$, then the super Leibniz identity can be expressed as $R_{[x, y]}=R_{y} R_{x}-(-1)^{\bar{i} \bar{j}} R_{x} R_{y}$ where $x \in L_{\bar{i}}, y \in L_{\bar{j}}$.

We denote by $R(L)$ the set of all right multiplication operators. It is not difficult to prove that $R(L)$ with the multiplication

$$
<R_{a}, R_{b}>:=R_{a} R_{b}-(-1)^{\bar{i} \bar{j}} R_{b} R_{a}
$$

for $R_{a} \in R(L)_{\bar{i}}, R_{b} \in R(L)_{\bar{j}}$, becomes a Lie superalgebra.

Note that the concepts of descending central sequence, the variety of Leibniz superalgebras and Engel's theorem are natural extensions from Lie theory.

Let $V=V_{\overline{0}} \oplus V_{\overline{1}}$ be the underlying vector space of $L, L=L_{\overline{0}} \oplus L_{\overline{1}} \in L e i b^{n, m}$, being $L e i b^{n, m}$ the variety of Leibniz superalgebras, and let $G(V)$ be the group of the invertible linear mappings of the form $f=f_{\overline{0}}+f_{\overline{1}}$, such that $f_{\overline{0}} \in G L(n, \mathbb{C})$ and $f_{\overline{1}} \in G L(m, \mathbb{C})$ (then $G(V)=G L(n, \mathbb{C}) \oplus G L(m, \mathbb{C})$ ). The action of $G(V)$ on $L e i b^{n, m}$ induces an action on the Leibniz superalgebras variety: two laws $\lambda_{1}, \lambda_{2}$ are isomorphic if there exists a linear mapping $f=f_{\overline{0}}+f_{\overline{1}} \in G(V)$, such that

$$
\lambda_{2}(x, y)=f_{\bar{i}+\bar{j}}^{-1}\left(\lambda_{1}\left(f_{\bar{i}}(x), f_{\bar{j}}(y)\right)\right), \text { for any } x \in V_{\bar{i}}, y \in V_{\bar{j}} .
$$

The description of the variety of any class of algebras or superalgebras is a difficult problem. Different papers (for example, $[4,9,20,21]$ ) are concerning the applications of algebraic groups theory to the description of the variety of Lie/Leibniz algebras.

Definition 2.2 For a Leibniz superalgebra $L=L_{\overline{0}} \oplus L_{\overline{1}}$ we define the right annihilator of $L$ as the set $\operatorname{Ann}(L):=\{x \in L:[L, x]=0\}$.

It is easy to see that $\operatorname{Ann}(L)$ is a two-sided ideal of $L$ and $[x, x] \in \operatorname{Ann}(L)$ for any $x \in L_{\overline{0}}$. This notion is nice and compatible with the right annihilator in Leibniz algebras. If we consider the ideal generated as $I:=$ ideal $<[x, y]+(-1)^{\bar{i} \bar{j}}[y, x]$ : $x \in L_{\bar{i}}, y \in L_{\bar{j}}>$, then $I \subset \operatorname{Ann}(L)$.

Let $L=L_{\overline{0}} \oplus L_{\overline{1}}$ be a nilpotent Leibniz superalgebra with $\operatorname{dim} L_{\overline{0}}=n$ and $\operatorname{dim} L_{\overline{1}}=m$. From Equation (2.1) we have that $R(L)$ is a Lie superalgebra, and in particular $R\left(L_{\overline{0}}\right)$ is a Lie algebra. As $L_{\overline{1}}$ has $L_{\overline{0}}$-module structure we can consider $R\left(L_{\overline{0}}\right)$ as a subset of $G L\left(V_{\overline{1}}\right)$, where $V_{\overline{1}}$ is the underlying vector space of $L_{\overline{1}}$. So, we have a Lie algebra formed by nilpotent endomorphisms of $V_{\overline{1}}$. Applying the Engel's theorem [22] we have the existence of a sequence of subspaces of $V_{\overline{1}}, V_{0} \subset V_{1} \subset$ $V_{2} \subset \cdots \subset V_{m}=V_{\overline{1}}$, with $R\left(L_{\overline{0}}\right)\left(V_{\overline{i+1}}\right) \subset V_{\bar{i}}$. Then, it can be defined the descending 
sequences $C^{k}\left(L_{\overline{0}}\right)$ and $C^{k}\left(L_{\overline{1}}\right)$ and the supernilindex in the same way as for Lie superalgebras.

Similar to the case of null-filiform Leibniz algebras, it is easy to check that a Leibniz superalgebra is null-filiform if and only if it is single generated. Moreover, a null-filiform superalgebra has maximal supernilindex (see [27]).

\section{Naturally Graded Lie and Leibniz Superalgebras}

In terms of algebras, recall that for nilpotent Lie and Leibniz algebras $\mathfrak{g}$, the descending central sequence defines a filtration over the algebra and therefore there is associated a graded Lie (resp. Leibniz) algebra structure that we denote as

$$
\operatorname{grg}:=\sum \mathcal{C}^{i-1}(\mathfrak{g}) / \mathcal{C}^{i}(\mathfrak{g})
$$

If $\mathfrak{g}, g r \mathfrak{g}$ are isomorphic, then $\mathfrak{g}$ is said to be naturally graded. In particular, there are only two non-isomorphic naturally graded filiform Lie algebras called $L_{n}$ and $Q_{n}$, whose laws can be expressed in an adapted basis $\left\{X_{0}, X_{1}, \ldots, X_{n}\right\}$ by the following nonzero bracket products (for more details it can be consulted [20,34])

$$
L_{n}:\left\{\left[X_{0}, X_{i}\right]=X_{i+1}, 1 \leq i \leq n-1 \quad Q_{n}: \begin{cases}{\left[X_{0}, X_{i}\right]=X_{i+1},} & 1 \leq i \leq n-1 \\ {\left[X_{i}, X_{n-i}\right]=(-1)^{i} X_{n},} & 1 \leq i \leq n-1\end{cases}\right.
$$

Note that both algebras are $(n+1)$-dimensional, although $Q_{n}$ only appears in the cases $n$ odd and $n \geq 5$.

Analogously, for superalgebra structures, i.e., nilpotent Lie and Leibniz superalgebras $\mathfrak{g}=\mathfrak{g}_{\overline{0}} \oplus \mathfrak{g}_{\overline{1}}$, can be considered the descending sequences $\mathcal{C}^{k}\left(\mathfrak{g}_{\bar{i}}\right):=\mathfrak{g}_{\bar{i}}$, $\mathcal{C}^{k+1}\left(\mathfrak{g}_{i}^{-}\right):=\left[\mathfrak{g}_{0}, \mathcal{C}^{k}\left(\mathfrak{g}_{i}^{-}\right)\right]$, with $k \geq 0, \bar{i} \in \mathbb{Z}_{2}$.

It can be seen that these sequences define a filtration over $\mathfrak{g}_{\overline{0}}$ and $\mathfrak{g}_{\overline{1}}$, respectively. Thus, we have on the one hand a structure of graded Lie (resp. Leibniz) algebra

$$
\operatorname{gr} \mathfrak{g}_{\overline{0}}=\sum \mathcal{C}^{i-1}\left(\mathfrak{g}_{\overline{0}}\right) / \mathcal{C}^{i}\left(\mathfrak{g}_{\overline{0}}\right)
$$

and on the other hand a structure of graded $\mathfrak{g}_{0}^{-}$-module

$$
\operatorname{gr} \mathfrak{g}_{\overline{1}}=\sum \mathcal{C}^{i-1}\left(\mathfrak{g}_{\overline{1}}\right) / \mathcal{C}^{i}\left(\mathfrak{g}_{\overline{1}}\right)
$$

If we denote $\mathfrak{g}_{\overline{0}}^{i}:=\mathcal{C}^{i-1}\left(\mathfrak{g}_{\overline{0}}\right) / \mathcal{C}^{i}\left(\mathfrak{g}_{0}\right)$ and $\mathfrak{g}_{\overline{1}}^{i}:=\mathcal{C}^{i-1}\left(\mathfrak{g}_{\overline{1}}\right) / \mathcal{C}^{i}\left(\mathfrak{g}_{\overline{1}}\right)$, then it is verified that

$$
\left[\mathfrak{g}_{\overline{0}}^{i}, \mathfrak{g}_{\overline{0}}^{j}\right] \subset \mathfrak{g}_{\overline{0}}^{i+j} \quad \text { and } \quad\left[\mathfrak{g}_{0}^{i}, \mathfrak{g}_{\overline{1}}^{j}\right] \subset \mathfrak{g}_{\overline{1}}^{i+j}
$$

Definition 3.1 Given a nilpotent Lie (resp. Leibniz) superalgebra $\mathfrak{g}=\mathfrak{g}_{0} \oplus \mathfrak{g}_{\overline{1}}$, consider $\mathfrak{g}^{i}=\mathfrak{g}_{0}^{i} \oplus \mathfrak{g}_{1}^{i}$, with $\mathfrak{g}_{\overline{0}}^{i}=\mathcal{C}^{i-1}\left(\mathfrak{g}_{\overline{0}}\right) / \mathcal{C}^{i}\left(\mathfrak{g}_{\overline{0}}\right)$ and $\mathfrak{g}_{1}^{i}=\mathcal{C}^{i-1}\left(\mathfrak{g}_{\overline{1}}\right) / \mathcal{C}^{i}\left(\mathfrak{g}_{\overline{1}}\right)$. Thus, $\mathfrak{g}$ is said to be naturally graded if the following conditions hold: 
1. $g r(\mathfrak{g})=\sum_{i \in \mathbb{N}} \mathfrak{g}^{i}$ is a graded Lie (resp. Leibniz) superalgebra $\left(\left[\mathfrak{g}^{i}, \mathfrak{g}^{j}\right] \subset \mathfrak{g}^{i+j}\right)$,

2. $\mathfrak{g}, \operatorname{gr}(\mathfrak{g})$ are isomorphic.

Note that when dealing with Lie superalgebras the usual bracket products [, ] are skew-symmetric and the products deriving from the symmetric pairing $S^{2} \mathfrak{g}_{\overline{1}} \longrightarrow \mathfrak{g}_{\overline{0}}$ and denoted by (, ) are symmetric.

Remark 3.1 Condition 1 is in fact required in the above definition. Note that in contrast with Lie or Leibniz algebras, $\operatorname{gr}(\mathfrak{g})$ could not be $\mathbb{Z}_{2}$-graded. Indeed, if we consider the nilpotent Leibniz superalgebra (which is also a Lie superalgebra) expressed in the adapted basis $\left\{X_{1}, X_{2}, X_{3}, X_{4}, Y_{1}\right\}$ as

$$
\left\{\begin{array}{l}
{\left[X_{2}, X_{1}\right]=X_{3}, \quad\left[X_{3}, X_{1}\right]=X_{4}, \quad\left[Y_{1}, Y_{1}\right]=X_{4}} \\
{\left[X_{1}, X_{2}\right]=-X_{3},\left[X_{1}, X_{3}\right]=-X_{4}}
\end{array}\right.
$$

we have $\mathfrak{g}^{1}=\mathfrak{g}_{0}^{1} \oplus \mathfrak{g}_{1}^{1}=\left(\mathcal{C}^{0}\left(\mathfrak{g}_{0}\right) / \mathcal{C}^{1}\left(\mathfrak{g}_{0}\right)\right) \oplus\left(\mathcal{C}^{0}\left(\mathfrak{g}_{\overline{1}}\right) / \mathcal{C}^{1}\left(\mathfrak{g}_{1}\right)\right)=<X_{1}, X_{2}>\oplus<$ $Y_{1}>$ Analogously, $L^{2}=<X_{3}>$ and $\mathfrak{g}^{3}=<X_{4}>$. Nevertheless, as $\left(Y_{1}, Y_{1}\right)=X_{4}$ then it is not verified that $\left[\mathfrak{g}^{1}, \mathfrak{g}^{1}\right] \subset \mathfrak{g}^{2}$ and consequently it is not graded.

\section{Naturally Graded Filiform Lie Superalgebras}

Among all the nilpotent Lie superalgebras, the filiform ones (those with maximal supernilindex) constitute one of the most important types due to its properties and applications. Thanks to both the theorem of adapted basis and the definition itself of filiform Lie superalgebras it can be seen that if $\mathfrak{g}=\mathfrak{g}_{\overline{0}} \oplus \mathfrak{g}_{\overline{1}}$ is a filiform Lie superalgebra, then its graded superalgebra associated $\operatorname{gr}(\mathfrak{g})=\sum_{i \in \mathbb{N}} \mathfrak{g}^{i}$ with $\left[\mathfrak{g}^{i}, \mathfrak{g}^{j}\right] \subset \mathfrak{g}^{i+j}$, is exactly

$$
\underbrace{<X_{0}, X_{1}, Y_{1}>}_{\mathfrak{g}^{1}} \oplus \underbrace{<X_{2}, Y_{2}>}_{\mathfrak{g}^{2}} \oplus \underbrace{<X_{3}, Y_{3}>}_{\mathfrak{g}^{3}} \oplus \cdots
$$

where for simplicity $\mathfrak{g}^{1}=\mathfrak{g}_{0}^{1} \oplus \mathfrak{g}_{1}^{1}=<X_{0}, X_{1}>\oplus<Y_{1}>$ has been replaced by $<X_{0}, X_{1}, Y_{1}>$ as there cannot be any possible confusion between even elements, called $X_{i}$, and odd ones, called $Y_{j}$. Similarly, the same replacement has been applied to every $\mathfrak{g}^{i}$. The last terms of $\operatorname{gr}(\mathfrak{g})$ depend on three possibilities:

1. If $n<m$, then

$$
\cdots \oplus \underbrace{<X_{n}, Y_{n}>}_{\mathfrak{g}^{n}} \oplus \underbrace{<Y_{n+1}>}_{\mathfrak{g}^{n+1}} \oplus \cdots \oplus \underbrace{<Y_{m}>}_{\mathfrak{g}^{m}}
$$

2. If $n=m$, then

$$
\cdots \oplus \underbrace{<X_{n-1}, Y_{n-1}>}_{\mathfrak{g}^{n-1}} \oplus \underbrace{<X_{n}, Y_{n}>}_{\mathfrak{g}^{n}}
$$


3. If $n>m$, then

$$
\cdots \oplus \underbrace{<X_{m}, Y_{m}>}_{\mathfrak{g}^{m}} \oplus \underbrace{<X_{m+1}>}_{\mathfrak{g}^{m+1}} \oplus \cdots \oplus \underbrace{<X_{n}>}_{\mathfrak{g}^{n}}
$$

Remark 4.1 It can be seen that the even elements of $\operatorname{gr}(\mathfrak{g})$, that is $\sum \mathfrak{g}_{\overline{0}}^{i}$, constitute a naturally graded filiform Lie algebra. Furthermore, the definition of naturally graded Lie superalgebras is a generalization of the one of naturally graded Lie algebras. Thus, if $\mathfrak{g}=\mathfrak{g}_{\overline{0}} \oplus \mathfrak{g}_{\overline{1}}$ is a naturally graded Lie superalgebra, then $\mathfrak{g}_{\overline{0}}$ is a naturally graded Lie algebra. Indeed, $\mathfrak{g}_{\overline{0}}^{i}$ is equal to $\mathcal{C}^{i-1}\left(\mathfrak{g}_{\overline{0}}\right) / \mathcal{C}^{i}\left(\mathfrak{g}_{\overline{0}}\right)$. Recall that the latter is used in the definition of naturally graded Lie algebras.

Thanks to this remark we can divide our study into two cases: either $\mathfrak{g}_{\overline{0}}=L_{n}$ or $\mathfrak{g}_{\overline{0}}=Q_{n}$. Nevertheless, before doing that we are going to obtain all the naturally graded filiform Lie superalgebras for low dimensions. We can accomplish such classification due to the results of [31]. The main result used in [31] is that any filiform Lie superalgebra is a linear deformation of the corresponding model filiform Lie superalgebra. Thus, it can be expressed by $L^{n, m}+\Psi$ with $L^{n, m}$ the law of the model filiform Lie superalgebra and $\Psi$ a linear (infinitesimal) deformation which is integrable that is verifying $\Psi \circ \Psi=0$. On the other hand, recall that an infinitesimal deformation of $L^{n, m}$ will be an element of the following space

$$
\begin{aligned}
Z_{0}^{2}\left(L^{n, m}, L^{n, m}\right)= & Z^{2}\left(L^{n, m}, L^{n, m}\right) \cap \operatorname{Hom}\left(\mathfrak{g}_{0} \wedge \mathfrak{g}_{0}, \mathfrak{g}_{0}\right) \oplus Z^{2}\left(L^{n, m}, L^{n, m}\right) \cap \operatorname{Hom}\left(\mathfrak{g}_{0} \wedge \mathfrak{g}_{1}, \mathfrak{g}_{1}\right) \oplus \\
& Z^{2}\left(L^{n, m}, L^{n, m}\right) \cap \operatorname{Hom}\left(S^{2} \mathfrak{g}_{1}, \mathfrak{g}_{0}\right)=A \oplus B \oplus C
\end{aligned}
$$

where $\mathfrak{g}_{0}=L_{0}^{n, m}$ and $\mathfrak{g}_{1}=L_{1}^{n, m}$. Recall also that all the infinitesimal deformations belonging to $C$ are always integrable in the aforementioned sense, for instance the infinitesimal deformations $\varphi_{i, j}$ which are determined from the symmetric product $\varphi_{i, j}\left(Y_{i}, Y_{i}\right)=X_{j}$. Thus, in particular the law $L^{n, m}+\varphi_{i, j}$ constitutes always a filiform Lie superalgebra.

Theorem 4.1 Let $\mathfrak{g}=\mathfrak{g}_{\overline{0}} \oplus \mathfrak{g}_{\overline{1}}$ be a non-degenerated filiform Lie superalgebra with $\operatorname{dim}\left(\mathfrak{g}_{0}\right)=n+1$ and $\operatorname{dim}\left(\mathfrak{g}_{1}^{-}\right)=m$. If $\operatorname{dim}(\mathfrak{g}) \leq 7$ and $\mathfrak{g}$ is naturally graded, then the law of $\mathfrak{g}$ will be isomorphic to one law of the following list.

\section{List of laws}

Pair of dimensions $n=2, m=1$. There is only one naturally graded filiform Lie superalgebra whose law can be expressed in an adapted basis by

$$
L^{2,1}+\varphi_{1,2}:\left\{\left[X_{0}, X_{1}\right]=X_{2},\left(Y_{1}, Y_{1}\right)=X_{2}\right.
$$

Pair of dimensions $n=2, m=2$. There is only one naturally graded filiform Lie superalgebra whose law can be expressed in an adapted basis as

$$
L^{2,2}+\varphi_{1,2}:\left\{\begin{array}{l}
{\left[X_{0}, X_{1}\right]=X_{2},\left(Y_{1}, Y_{1}\right)=X_{2}} \\
{\left[X_{0}, Y_{1}\right]=Y_{2},}
\end{array}\right.
$$


Pair of dimensions $n=2, m=3$. There is only one naturally graded filiform Lie superalgebra whose law can be expressed in an adapted basis by

$$
L^{2,3}+\varphi_{1,2}:\left\{\begin{array}{l}
{\left[X_{0}, X_{1}\right]=X_{2}, \quad\left(Y_{1}, Y_{1}\right)=X_{2}} \\
{\left[X_{0}, Y_{i}\right]=Y_{i+1}, 1 \leq i \leq 2}
\end{array}\right.
$$

Pair of dimensions $n=2, m=4$. There is only one naturally graded filiform Lie superalgebra whose law can be expressed in an adapted basis by

$$
L^{2,4}+\varphi_{1,2}:\left\{\begin{array}{l}
{\left[X_{0}, X_{1}\right]=X_{2},\left[X_{0}, Y_{i}\right]=Y_{i+1}, 1 \leq i \leq 3} \\
\left(Y_{1}, Y_{1}\right)=X_{2}
\end{array}\right.
$$

Pair of dimensions $n=3, m=2$. There are two non-isomorphic naturally graded filiform Lie superalgebras whose laws can be expressed in an adapted basis by

$$
\begin{aligned}
& L^{3,2}+\varphi_{1,2}: \\
& \left\{\begin{array}{ll}
{\left[X_{0}, X_{i}\right]=X_{i+1}, 1 \leq i \leq 2\left(Y_{1}, Y_{1}\right)=X_{2}} \\
{\left[X_{0}, Y_{1}\right]=Y_{2}}
\end{array} \quad \begin{array}{l}
{\left[X_{0}, X_{i}\right]=X_{i+1}, 1 \leq i \leq 2\left(Y_{1}, Y_{1}\right)=X_{2}} \\
{\left[X_{0}, Y_{1}\right]=Y_{2}} \\
{\left[X_{1}, Y_{1}\right]=Y_{2}}
\end{array}\right. \\
&
\end{aligned}
$$

Pair of dimensions $n=4, m=2$. There is only one naturally graded filiform Lie superalgebra whose law can be expressed in an adapted basis by

$$
L^{4,2}+\bar{\varphi}_{2,4}:\left\{\begin{array}{lr}
{\left[X_{0}, X_{i}\right]=X_{i+1}, 1 \leq i \leq 3} & \left(Y_{1}, Y_{2}\right)=X_{3} \\
{\left[X_{0}, Y_{1}\right]=Y_{2}} & \left(Y_{2}, Y_{2}\right)=X_{4} \\
\left(Y_{1}, Y_{1}\right)=2 X_{2} &
\end{array}\right.
$$

Pair of dimensions $m=3, n=3$. There are two non-isomorphic naturally graded filiform Lie superalgebras whose laws can be expressed in an adapted basis as

$$
\begin{aligned}
& L^{3,3}+\varphi_{1,2}: \\
& \left\{\begin{array} { l } 
{ [ X _ { 0 } , X _ { i } ] = X _ { i + 1 } , 1 \leq i \leq 2 ( Y _ { 1 } , Y _ { 1 } ) = X _ { 2 } } \\
{ [ X _ { 0 } , Y _ { j } ] = Y _ { j + 1 } , 1 \leq j \leq 2 ( Y _ { 1 } , Y _ { 2 } ) = \frac { 1 } { 2 } X _ { 3 } }
\end{array} \left\{\begin{array}{l}
{\left[X_{0}, X_{i}\right]=X_{i+1}, 1 \leq i \leq 2\left(Y_{1}, Y_{1}\right)=X_{2}} \\
{\left[X_{0}, Y_{j}\right]=Y_{j+1}, 1 \leq j \leq 2\left(Y_{1}, Y_{2}\right)=\frac{1}{2} X_{3}} \\
{\left[X_{1}, Y_{1}\right]=Y_{2}} \\
{\left[X_{1}, Y_{2}\right]=Y_{3}}
\end{array}\right.\right.
\end{aligned}
$$

Proof In [31], Theorem 8.1 (Classification's theorem) pages 80-81 is obtained the classification up to isomorphism of all the non-degenerated filiform Lie superalgebras with total dimension less or equal to 7. From this list we check which ones are naturally graded obtaining then the result of the statement of this theorem.

Remark 4.2 There is no any naturally graded filiform Lie superalgebra for the cases $n=3, m=1 ; n=4, m=1$ and $n=5, m=1$. Note also that all the naturally graded filiform Lie superalgebras of the aforementioned theorem derive from the naturally graded filiform Lie algebra $L_{n}$. There is no one deriving from $Q_{n}$.

Next, we start with $\operatorname{dim}\left(\mathfrak{g}_{\overline{0}}\right)=3=n+1$. 


\section{1 $n=2$ and $m$ Arbitrary}

Throughout this subsection we are going to study naturally graded filiform Lie superalgebras $\mathfrak{g}=\mathfrak{g}_{\overline{0}} \oplus \mathfrak{g}_{\overline{1}}$ with $\operatorname{dim}\left(\mathfrak{g}_{0}\right)=n+1=3$ and $m \geq 5$ since the cases $1 \leq m \leq 4$ have already been studied.

Theorem 4.2 Let $\mathfrak{g}=\mathfrak{g}_{\overline{0}} \oplus \mathfrak{g}_{\overline{1}}$ be a non-degenerated filiform Lie superalgebra with $\operatorname{dim}\left(\mathfrak{g}_{\overline{0}}\right)=3$ and $\operatorname{dim}\left(\mathfrak{g}_{\overline{1}}\right)=m$ with $m \geq 5$. If $\mathfrak{g}$ is naturally graded, then the law of $\mathfrak{g}$ is isomorphic to

$$
L^{2, m}+\varphi_{1,2}: \begin{cases}{\left[X_{0}, X_{1}\right]=X_{2}} & \left(Y_{1}, Y_{1}\right)=X_{2} \\ {\left[X_{0}, Y_{i}\right]=Y_{i+1},} & 1 \leq i \leq m-1\end{cases}
$$

Proof It can be seen that $\mathfrak{g}_{\overline{0}}=L_{2}$ since $\operatorname{dim}\left(\mathfrak{g}_{\overline{0}}\right)=3$ and $\mathfrak{g}_{\overline{0}}$ is a naturally graded filiform Lie algebra. Therefore $g r(\mathfrak{g})$ is exactly

$$
\underbrace{<X_{0}, X_{1}, Y_{1}>}_{\mathfrak{g}^{1}} \oplus \underbrace{<X_{2}, Y_{2}>}_{\mathfrak{g}^{2}} \oplus \underbrace{<Y_{3}>}_{\mathfrak{g}^{3}} \oplus \cdots \oplus \underbrace{<Y_{m}>}_{\mathfrak{g}^{m}}
$$

Thus, the bracket products can be considered to be

$$
\left\{\begin{array}{l}
{\left[X_{0}, X_{1}\right]=X_{2}, \quad\left[X_{1}, Y_{i}\right]=b_{1 i} Y_{i+1}, 1 \leq i \leq m-1} \\
{\left[X_{0}, Y_{i}\right]=Y_{i+1}, 1 \leq i \leq m-1,\left[X_{2}, Y_{i}\right]=b_{2 i} Y_{i+2}, 1 \leq i \leq m-2} \\
\left(Y_{1}, Y_{1}\right)=c_{11} X_{2}
\end{array}\right.
$$

with $c_{11} \neq 0$ in order not to have a degenerated Lie superalgebra, that is, a Lie algebra. By means of a simple change of scale it can be seen that there is no loss of generality in considering $c_{11}=1$. It can be seen that $\left(Y_{1}, Y_{1}\right)=X_{2}$ corresponds to the cocycle $\varphi_{1,2}$. Additionally, the remaining structure constants $b_{1 i}, b_{2 i}$ indeed will be determined by the cocycles $\Psi_{1,1}^{2}, \Psi_{2,1}^{3}$, respectively (more details can be consulted [25]), given as

$$
\Psi_{1,1}^{2}:\left[X_{1}, Y_{j}\right]=Y_{j+1}, 1 \leq j \leq m-1 \quad \Psi_{2,1}^{3}:\left\{\begin{array}{l}
{\left[X_{1}, Y_{j}\right]=-(j-1) Y_{j+1}, 2 \leq j \leq m-1} \\
{\left[X_{2}, Y_{j}\right]=Y_{j+2}, 1 \leq j \leq m-2}
\end{array}\right.
$$

These cocycles, or so-called infinitesimal deformations, are the only one "naturally graded," that is, of weight 0 . Recall that the weight of $\Psi_{k, 1}^{s}$ is $s-k-1$.

Next, we apply the method of classification used in [31]: every filiform Lie superalgebra can be expressed by $L^{n, m}+\Psi$, with $L^{n, m}$ the law of the model filiform Lie superalgebra and $\Psi$ a linear (infinitesimal) deformation that verifies $\Psi \circ \Psi=0$, being $\Psi \circ \Psi(x, y, z)=\Psi(\Psi(x, y), z)+\Psi(\Psi(z, x), y)+\Psi(\Psi(y, z), x)$. Thus, we can consider

$$
L^{2, m}+\Psi: \begin{cases}{\left[X_{0}, X_{1}\right]=X_{2},} & {\left[X_{1}, Y_{j}\right]=[a-b(j-1)] Y_{j+1}, 2 \leq i \leq m-1} \\ {\left[X_{0}, Y_{i}\right]=Y_{i+1}, \quad 1 \leq i \leq m-1,} & {\left[X_{2}, Y_{j}\right]=b Y_{j+2}, 1 \leq i \leq m-2} \\ {\left[X_{1}, Y_{1}\right]=a Y_{2},} & \left(Y_{1}, Y_{1}\right)=X_{2}\end{cases}
$$


where $\Psi=\varphi_{1,2}+a \Psi_{1,1}^{2}+b \Psi_{2,1}^{3}$. From the condition $\Psi \circ \Psi\left(X_{1}, X_{2}, Y_{1}\right)=0$ it is obtained that $b=0$. Then applying the isomorphism, or so-called change of basis, defined by $X_{0}^{\prime}=X_{0}, X_{1}^{\prime}=X_{1}-a X_{0}, X_{2}^{\prime}=X_{2}$ and $Y_{j}^{\prime}=Y_{j}$ for any $j \in \mathbb{N}$, it can be seen that there is no loss of generality in supposing $a=0$. Consequently, it remains only $L^{2, m}+\varphi_{1,2}$.

\section{$4.2 n=3$ and $m=4$}

In this case we have the following results:

Theorem 4.3 Let $\mathfrak{g}=\mathfrak{g}_{\overline{0}} \oplus \mathfrak{g}_{\overline{1}}$ be a non-degenerated filiform Lie superalgebra with $\operatorname{dim}\left(\mathfrak{g}_{\overline{0}}\right)=\operatorname{dim}\left(\mathfrak{g}_{\overline{1}}\right)=4$. If $\mathfrak{g}$ is naturally graded, then the law of $\mathfrak{g}$ is isomorphic to one of the following lists of pairwise non-isomorphic laws

$$
\begin{array}{cll}
L^{3,4}+\varphi_{1,2}: & L^{3,4}+\varphi_{1,2}+\Psi_{3,1}^{4}: & L^{3,4}+\varphi_{1,2}+\Psi_{1,1}^{2}: \\
\left\{\begin{array}{l}
{\left[X_{0}, X_{i}\right]=X_{i+1}} \\
{\left[X_{0}, Y_{j}\right]=Y_{j+1}} \\
\left(Y_{1}, Y_{1}\right)=X_{2} \\
\left(Y_{1}, Y_{2}\right)=\frac{1}{2} X_{3}
\end{array} \quad \begin{array}{l}
{\left[X_{0}, X_{i}\right]=X_{i+1}\left(Y_{1}, Y_{1}\right)=X_{2}} \\
\left.\left[X_{0}, Y_{j}\right]=Y_{j+1}\left(X_{1}, Y_{2}\right]=Y_{4}\right) \\
{\left[X_{2}, Y_{2}\right]=-Y_{4}} \\
{\left[X_{3}, Y_{1}\right]=Y_{4}}
\end{array}\right. & \left\{\begin{array}{l}
{\left[X_{0}, X_{i}\right]=X_{i+1}} \\
{\left[X_{0}, Y_{j}\right]=Y_{j+1}} \\
{\left[X_{1}, Y_{j}\right]=Y_{j+1}} \\
\left(Y_{1}, Y_{1}\right)=X_{2} \\
\left(Y_{1}, Y_{2}\right)=\frac{1}{2} X_{3}
\end{array}\right.
\end{array}
$$

with $1 \leq i \leq 2$ and $1 \leq j \leq 3$.

Proof We consider naturally graded filiform Lie superalgebras $\mathfrak{g}=\mathfrak{g}_{\overline{0}} \oplus \mathfrak{g}_{\overline{1}}$ with $\operatorname{dim}\left(\mathfrak{g}_{0}\right)=4$ and $\operatorname{dim}\left(\mathfrak{g}_{\overline{1}}\right)=4$. Therefore $\mathfrak{g}_{0}=L_{3}$ and $\operatorname{gr}(\mathfrak{g})$ is

$$
\underbrace{<X_{0}, X_{1}, Y_{1}>}_{\mathfrak{g}^{1}} \oplus \underbrace{<X_{2}, Y_{2}>}_{\mathfrak{g}^{2}} \oplus \underbrace{<X_{3}, Y_{3}>}_{\mathfrak{g}^{3}} \oplus \underbrace{<Y_{4}>}_{\mathfrak{g}^{4}}
$$

Thus, the bracket products can be considered to be

$$
\begin{cases}{\left[X_{0}, X_{i}\right]=X_{i+1}, i=1,2} & {\left[X_{3}, Y_{1}\right]=b_{31} Y_{4}} \\ {\left[X_{0}, Y_{i}\right]=Y_{i+1}, 1 \leq i \leq 3} & \left(Y_{1}, Y_{1}\right)=c_{11} X_{2} \\ {\left[X_{1}, Y_{i}\right]=b_{1 i} Y_{i+1}, 1 \leq i \leq 3} & \left(Y_{1}, Y_{2}\right)=c_{12} X_{3} \\ {\left[X_{2}, Y_{i}\right]=b_{2 i} Y_{i+2}, 1 \leq i \leq 2} & \end{cases}
$$

In a similar way to the previous case the structure constants can be considered to be determined by the cocycles $\Psi_{1,1}^{2}, \Psi_{2,1}^{3}, \Psi_{3,1}^{4}$ and $\varphi_{1,2}$ (see [8,25]). In general, $\Psi_{1,1}^{2}$ and $\Psi_{2,1}^{3}$ have already been described in the above case, and $\Psi_{3,1}^{4}$ and $\varphi_{1,2}$ are as follows

$$
\Psi_{3,1}^{4}:\left\{\begin{array}{l}
{\left[X_{1}, Y_{3}\right]=Y_{4}} \\
{\left[X_{2}, Y_{2}\right]=-Y_{4}} \\
{\left[X_{3}, Y_{1}\right]=Y_{4}}
\end{array} \quad \varphi_{1,2}:\left\{\begin{array}{l}
\left(Y_{1}, Y_{1}\right)=X_{2} \\
\left(Y_{1}, Y_{2}\right)=\frac{1}{2} X_{3}
\end{array}\right.\right.
$$


Thus, in order not to have a degenerated case, that is, a Lie algebra, the coefficient of $\varphi_{1,2}$ must be different from zero and then, it can be easily seen that there is no loss of generality in considering this coefficient equals 1 . Therefore we can consider

$$
L^{3,4}+\Psi: \begin{cases}{\left[X_{0}, X_{1}\right]=X_{2},} & {\left[X_{2}, Y_{1}\right]=a_{2} Y_{3}} \\ {\left[X_{0}, X_{2}\right]=X_{3},} & {\left[X_{2}, Y_{2}\right]=\left(a_{2}-a_{3}\right) Y_{4}} \\ {\left[X_{0}, Y_{i}\right]=Y_{i+1}, 1 \leq i \leq 3,} & {\left[X_{3}, Y_{1}\right]=a_{3} Y_{4}} \\ {\left[X_{1}, Y_{1}\right]=a_{1} Y_{2},} & \left(Y_{1}, Y_{1}\right)=X_{2} \\ {\left[X_{1}, Y_{2}\right]=\left(a_{1}-a_{2}\right) Y_{3},} & \left(Y_{1}, Y_{2}\right)=\frac{1}{2} X_{3} \\ {\left[X_{1}, Y_{3}\right]=\left(a_{1}-2 a_{2}+a_{3}\right) Y_{4},} & \end{cases}
$$

being $\Psi=\varphi_{1,2}+a_{1} \Psi_{1,1}^{2}+a_{2} \Psi_{2,1}^{3}+a_{3} \Psi_{3,1}^{4}$. From the condition $\Psi \circ \Psi\left(Y_{1}, Y_{1}, Y_{1}\right)=0$ we obtain $a_{2}=0$, and on account of $\Psi \circ \Psi\left(X_{1}, X_{2}, Y_{1}\right)=0$ we conclude $a_{1} a_{3}=0$.

- If $a_{1}=0$ we distinguish two cases: $a_{3}=0$ or $a_{3} \neq 0$.

- In the case of $a_{3}=0$ we obtain $L^{3,4}+\varphi_{1,2}$.

- If $a_{3} \neq 0$ without loss of generality we can consider $a_{3}=1$ (by means of a simple change of scale) obtaining $L^{3,4}+\varphi_{1,2}+\Psi_{3,1}^{4}$.

- If $a_{1} \neq 0$ then $a_{3}=0$. Therefore, applying the isomorphism (change of scale) defined by $X_{0}^{\prime}=a_{1} X_{0}, X_{1}^{\prime}=X_{1}, X_{2}^{\prime}=a_{1} X_{2}, X_{3}^{\prime}=\left(a_{1}\right)^{2} X_{3}, Y_{1}^{\prime}=\sqrt{a_{1}} Y_{1}$, $Y_{2}^{\prime}=a_{1} \sqrt{a_{1}} Y_{2}$ and $Y_{3}^{\prime}=\left(a_{1}\right)^{2} \sqrt{a_{1}} Y_{3}$, it can be seen that there is no loss of generality in supposing $a_{1}=1$, and we obtain $L^{3,4}+\varphi_{1,2}+\Psi_{1,1}^{2}$.

\section{$4.3 n=3$ and $m \geq 5$}

Throughout this subsection we are going to study naturally graded filiform Lie superalgebras $\mathfrak{g}=\mathfrak{g}_{\overline{0}} \oplus \mathfrak{g}_{\overline{1}}$ with $\operatorname{dim}\left(\mathfrak{g}_{\overline{0}}\right)=n+1=4$ and $m \geq 5$ since the cases $1 \leq m \leq 4$ have already been studied.

Theorem 4.4 Let $\mathfrak{g}=\mathfrak{g}_{0} \oplus \mathfrak{g}_{1}$ be a non-degenerated filiform Lie superalgebra with $\operatorname{dim}\left(\mathfrak{g}_{\overline{0}}\right)=4$ and $\operatorname{dim}\left(\mathfrak{g}_{\overline{1}}\right)=m$ with $m \geq 5$. If $\mathfrak{g}$ is naturally graded then the law of $\mathfrak{g}$ is isomorphic to one of the two non-isomorphic laws:

$$
L^{3, m}+\varphi_{1,2}:\left\{\begin{array}{l}
{\left[X_{0}, X_{i}\right]=X_{i+1}} \\
{\left[X_{0}, Y_{j}\right]=Y_{j+1}} \\
\left(Y_{1}, Y_{1}\right)=X_{2} \\
\left(Y_{1}, Y_{2}\right)=\frac{1}{2} X_{3}
\end{array} L^{3, m}+\varphi_{1,2}+\Psi_{1,1}^{2}:\left\{\begin{array}{l}
{\left[X_{0}, X_{i}\right]=X_{i+1}\left(Y_{1}, Y_{1}\right)=X_{2}} \\
{\left[X_{0}, Y_{j}\right]=Y_{j+1}\left(Y_{1}, Y_{2}\right)=\frac{1}{2} X_{3}} \\
{\left[X_{1}, Y_{j}\right]=Y_{j+1}}
\end{array}\right.\right.
$$

with $1 \leq i \leq 2,1 \leq j \leq m-1$.

Proof Let $\mathfrak{g}=\mathfrak{g}_{\overline{0}} \oplus \mathfrak{g}_{\overline{1}}$ be a naturally graded filiform Lie superalgebra with $\operatorname{dim}\left(\mathfrak{g}_{\overline{0}}\right)=$ 4 and $\operatorname{dim}\left(\mathfrak{g}_{1}\right)=m \geq 5$. Therefore, $\mathfrak{g}_{0}=L_{4}$ and $\operatorname{gr}(\mathfrak{g})$ is

$$
\underbrace{<X_{0}, X_{1}, Y_{1}>}_{\mathfrak{g}^{1}} \oplus \underbrace{<X_{2}, Y_{2}>}_{\mathfrak{g}^{2}} \oplus \underbrace{<X_{3}, Y_{3}>}_{\mathfrak{g}^{3}} \oplus \underbrace{<Y_{4}>}_{\mathfrak{g}^{4}} \oplus \cdots \oplus \underbrace{<Y_{m}>}_{\mathfrak{g}^{m}}
$$


So the bracket products are

$$
\begin{array}{ll}
{\left[X_{0}, X_{1}\right]=X_{2},} & {\left[X_{2}, Y_{i}\right]=b_{2 i} Y_{i+2}, 1 \leq i \leq m-2} \\
{\left[X_{0}, X_{2}\right]=X_{3},} & {\left[X_{3}, Y_{i}\right]=b_{3 i} Y_{i+3}, 1 \leq i \leq m-3} \\
{\left[X_{0}, Y_{i}\right]=Y_{i+1}, 1 \leq i \leq m-1,} & \left(Y_{1}, Y_{1}\right)=c_{11} X_{2} \\
{\left[X_{1}, Y_{i}\right]=b_{1 i} Y_{i+1}, 1 \leq i \leq m-1,} & \left(Y_{1}, Y_{2}\right)=c_{12} X_{3}
\end{array}
$$

As in the above case and in order not to have any degenerated case the coefficient of $\varphi_{1,2}$ must be different from zero and then, it can be easily seen that there is no loss of generality in considering this coefficient equal to 1 . Therefore we can consider $L^{3, m}+\Psi$, with $\Psi=\varphi_{1,2}+a_{1} \Psi_{1,1}^{2}+a_{2} \Psi_{2,1}^{3}+a_{3} \Psi_{3,1}^{4}$, and we have the following products:

$$
L^{3, m}+\Psi:\left\{\begin{array}{l}
{\left[X_{0}, X_{1}\right]=X_{2}} \\
{\left[X_{0}, X_{2}\right]=X_{3}} \\
{\left[X_{0}, Y_{i}\right]=Y_{i+1}, 1 \leq i \leq m-1} \\
{\left[X_{1}, Y_{1}\right]=a_{1} Y_{2},} \\
{\left[X_{1}, Y_{i}\right]=\left(a_{1}-(i-1) a_{2}+\frac{(i-1)(i-2)}{2} a_{3}\right) Y_{i+1}, 2 \leq i \leq m-1} \\
{\left[X_{2}, Y_{i}\right]=\left(a_{2}-(i-1) a_{3}\right) Y_{i+2}, 1 \leq i \leq m-2} \\
{\left[X_{3}, Y_{i}\right]=a_{3} Y_{i+3}, 1 \leq i \leq m-3} \\
\left(Y_{1}, Y_{1}\right)=X_{2} \\
\left(Y_{1}, Y_{2}\right)=\frac{1}{2} X_{3}
\end{array}\right.
$$

The condition $\Psi \circ \Psi\left(Y_{1}, Y_{1}, Y_{1}\right)=0$ leads to $a_{2}=0$, and then, from the condition $\Psi \circ \Psi\left(X_{1}, X_{3}, Y_{1}\right)=0$ it is obtained that $a_{3}=0$. So if $a_{1}=0$ we have $L^{3, m}+\varphi_{1,2}$. Otherwise we have $L^{3,4}+\varphi_{1,2}+\Psi_{1,1}^{2}$ after applying a simple change of scale.

\section{$4.4 n$ and $m$ Arbitrary with $n>2 m$}

Theorem 4.5 There is not any non-degenerated naturally graded filiform Lie superalgebra $\mathfrak{g}=\mathfrak{g}_{0} \oplus \mathfrak{g}_{\overline{1}}$ with the condition $n>2 m$, being $\operatorname{dim}\left(\mathfrak{g}_{0}\right)=n+1$ and $\operatorname{dim}\left(\mathfrak{g}_{\overline{1}}\right)=m$.

Proof This result derives from the fact that there is not any cocycle $\varphi_{k, s}$ naturally graded, that is, of weight equals 0 since we have always $\max \left\{\frac{n-2 m-1}{2}, n-2 m\right\} \leq$ weight $\left(\varphi_{k, s}\right)$ (see [8], Proposition 6.4).

Remark 4.3 We have as particular cases of the above theorem $m=1, n \geq 3$; $m=$ $2, n \geq 5$ and $m=3, n \geq 7$. Consequently, and in order to study all the possibilities for $m \leq 3$, it only remains to consider the cases $4 \leq n \leq 6$ and $m=3$.

\section{$4.5 n=4$ and $m=3$}

Theorem 4.6 Let $\mathfrak{g}=\mathfrak{g}_{\overline{0}} \oplus \mathfrak{g}_{\overline{1}}$ be a non-degenerated filiform Lie superalgebra with $\operatorname{dim}\left(\mathfrak{g}_{\overline{0}}\right)=5$ and $\operatorname{dim}\left(\mathfrak{g}_{\overline{1}}\right)=3$. If $\mathfrak{g}$ is naturally graded, then the law of $\mathfrak{g}$ is isomorphic to one of the following non-isomorphic laws: 


$$
\begin{aligned}
& L^{4,3}+\varphi_{2,4}: \\
& L^{4,3}+\varphi_{2,4}+\Psi_{1,1}^{2}+2 \Psi_{2,1}^{3}: \\
& \left\{\begin{array} { l } 
{ [ X _ { 0 } , X _ { i } ] = X _ { i + 1 } ( Y _ { 1 } , Y _ { 3 } ) = - X _ { 4 } } \\
{ [ X _ { 0 } , Y _ { j } ] = Y _ { j + 1 } ( Y _ { 2 } , Y _ { 2 } ) = X _ { 4 } }
\end{array} \left\{\begin{array}{ll}
{\left[X_{0}, X_{i}\right]=X_{i+1},} & {\left[X_{2}, Y_{1}\right]=Y_{3}} \\
{\left[X_{0}, Y_{j}\right]=Y_{j+1},} & \left(Y_{1}, Y_{3}\right)=-X_{4} \\
{\left[X_{1}, Y_{1}\right]=Y_{2}} & \left(Y_{2}, Y_{2}\right)=X_{4} \\
{\left[X_{1}, Y_{2}\right]=-Y_{3}} &
\end{array}\right.\right. \\
& L^{4,3}+\varphi_{1,2}+t \varphi_{2,4}: \\
& \left\{\begin{array} { l } 
{ [ X _ { 0 } , X _ { i } ] = X _ { i + 1 } , ( Y _ { 1 } , Y _ { 2 } ) = \frac { 1 } { 2 } X _ { 3 } } \\
{ [ X _ { 0 } , Y _ { j } ] = Y _ { j + 1 } , } \\
{ ( Y _ { 1 } , Y _ { 1 } ) = X _ { 2 } , \quad ( Y _ { 2 } , Y _ { 2 } ) = t \frac { 1 } { 2 } - t ) X _ { 4 } }
\end{array} \quad \left\{\begin{array}{ll}
{\left[X_{0}, X_{i}\right]=X_{i+1},} & \left(Y_{1}, Y_{1}\right)=4 X_{2} \\
{\left[X_{0}, Y_{j}\right]=Y_{j+1},} & \left(Y_{1}, Y_{2}\right)=2 X_{3} \\
{\left[X_{1}, Y_{1}\right]=Y_{2},} & \left(Y_{1}, Y_{3}\right)=X_{4} \\
{\left[X_{1}, Y_{2}\right]=Y_{3},} & \left(Y_{2}, Y_{2}\right)=X_{4}
\end{array}\right.\right.
\end{aligned}
$$

with $1 \leq i \leq 3,1 \leq j \leq 2$ and $t \in \mathbb{C}$ a parameter.

Proof Let $\mathfrak{g}=\mathfrak{g}_{0} \oplus \mathfrak{g}_{1}$ be a naturally graded filiform Lie superalgebra with $\operatorname{dim}\left(\mathfrak{g}_{0}\right)=$ 5 and $\operatorname{dim}\left(\mathfrak{g}_{\overline{1}}\right)=3$. Therefore, $\mathfrak{g}_{\overline{0}}=L_{4}$ and $\operatorname{gr}(\mathfrak{g})$ is

$$
\underbrace{<X_{0}, X_{1}, Y_{1}>}_{\mathfrak{g}^{1}} \oplus \underbrace{<X_{2}, Y_{2}>}_{\mathfrak{g}^{2}} \oplus \underbrace{<X_{3}, Y_{3}>}_{\mathfrak{g}^{3}} \oplus \underbrace{<X_{4}>}_{\mathfrak{g}^{4}}
$$

In an analogous way to previous cases, we can consider $L^{4,3}+\Psi$, with $\Psi=$ $c \varphi_{1,2}+d \varphi_{2,4}+a_{1} \Psi_{1,1}^{2}+a_{2} \Psi_{2,1}^{3}$, and we have the following products:

$$
L^{3,4}+\Psi: \begin{cases}{\left[X_{0}, X_{i}\right]=X_{i+1}, 1 \leq i \leq 3,} & \left(Y_{1}, Y_{1}\right)=c X_{2} \\ {\left[X_{0}, Y_{i}\right]=Y_{i+1}, 1 \leq i \leq 2,} & \left(Y_{1}, Y_{2}\right)=\frac{c}{2} X_{3} \\ {\left[X_{1}, Y_{1}\right]=a_{1} Y_{2},} & \left(Y_{1}, Y_{3}\right)=\left(\frac{c}{2}-d\right) X_{4} \\ {\left[X_{1}, Y_{2}\right]=\left(a_{1}-a_{2}\right) Y_{3},} & \left(Y_{2}, Y_{2}\right)=d X_{4} \\ {\left[X_{2}, Y_{1}\right]=a_{2} Y_{3}} & \end{cases}
$$

where at least $c$ or $d$ is nonzero. The condition $\Psi \circ \Psi\left(Y_{1}, Y_{1}, Y_{1}\right)=0$ gives us $a_{2} c=0$, and since $\Psi \circ \Psi\left(X_{1}, Y_{1}, Y_{2}\right)=0$, we have $a_{1} d+\left(a_{2}-a_{1}\right)\left(\frac{c}{2}-d\right)=0$.

- If $c=0$ then $d \neq 0$, and from $a_{1} d+\left(a_{2}-a_{1}\right)\left(\frac{c}{2}-d\right)=0$ we have $a_{2}=2 a_{1}$.

- For $a_{1}=0$ is $a_{2}=0$ and $d \neq 0$, so using a change of scale $d=1$ and we obtain $L^{4,3}+\varphi_{2,4}$.

- For $a_{1} \neq 0$ again $d \neq 0$, and with a similar change of scale $a_{1}=d=1$ and $a_{2}=2$, obtaining $L^{4,3}+\varphi_{2,4}+\Psi_{1,1}^{2}+2 \Psi_{2,1}^{3}$.

- In the case of $c \neq 0$ then necessarily $a_{2}=0$ and from $a_{1} d+\left(a_{2}-a_{1}\right)\left(\frac{c}{2}-d\right)=0$ is $4 a_{1} d=a_{1} c$.

- For $a_{1}=0$ by means of the change of scale $c=1, d=t$, being $t$ a parameter, we obtain $L^{4,3}+\varphi_{1,2}+t \varphi 2,4$.

- For $a_{1} \neq 0$ is $d \neq 0$ and with the change of scale $a_{1}=d=1, a_{2}=0$ and $c=4$ we obtain $L^{4,3}+4 \varphi_{1,2}+\varphi_{2,4}+\Psi_{1,1}^{2}$. 


\section{$4.6 n=5$ and $m=3$}

Theorem 4.7 Let $\mathfrak{g}=\mathfrak{g}_{0} \oplus \mathfrak{g}_{1}$ be a non-degenerated filiform Lie superalgebra with $\operatorname{dim}\left(\mathfrak{g}_{\overline{0}}\right)=6$ and $\operatorname{dim}\left(\mathfrak{g}_{\overline{1}}\right)=3$. If $\mathfrak{g}$ is naturally graded, then the law of $\mathfrak{g}$ will be isomorphic to the following law

$$
L^{5,3}+\bar{\varphi}_{2,4}: \begin{cases}{\left[X_{0}, X_{i}\right]=X_{i+1}, 1 \leq i \leq 4,} & \left(Y_{1}, Y_{3}\right)=\frac{1}{2} X_{4} \\ {\left[X_{0}, Y_{i}\right]=Y_{i+1}, 1 \leq i \leq 2,} & \left(Y_{2}, Y_{2}\right)=X_{4} \\ \left(Y_{1}, Y_{1}\right)=3 X_{2}, & \left(Y_{2}, Y_{3}\right)=\frac{1}{2} X_{5} \\ \left(Y_{1}, Y_{2}\right)=\frac{3}{2} X_{3}, & \end{cases}
$$

Proof Let $\mathfrak{g}=\mathfrak{g}_{0} \oplus \mathfrak{g}_{\overline{1}}$ be a naturally graded filiform Lie superalgebra with $\operatorname{dim}\left(\mathfrak{g}_{0}\right)=$ 6 and $\operatorname{dim}\left(\mathfrak{g}_{1}\right)=3$. Then $\operatorname{gr}(\mathfrak{g})$ is

$$
\underbrace{<X_{0}, X_{1}, Y_{1}>}_{\mathfrak{g}^{1}} \oplus \underbrace{<X_{2}, Y_{2}>}_{\mathfrak{g}^{2}} \oplus \underbrace{<X_{3}, Y_{3}>}_{\mathfrak{g}^{3}} \oplus \underbrace{<X_{4}>}_{\mathfrak{g}^{4}} \oplus \underbrace{<X_{5}>}_{\mathfrak{g}^{5}}
$$

In this case we have to distinguish two separate sub-cases: $\mathfrak{g}_{\overline{0}}=L_{5}$ or $\mathfrak{g}_{\overline{0}}=Q_{5}$, the two possible naturally graded filiform Lie algebras for this dimension.

- If $\mathfrak{g}_{\overline{0}}=L_{5}$, and as in previous cases we get $L^{5,3}+\Psi$, with $\Psi=c \bar{\varphi}_{2,4}+a_{1} \Psi_{1,1}^{2}+$ $a_{2} \Psi_{2,1}^{3}$, being $\bar{\varphi}_{2,4}=3 \varphi_{1,2}+\varphi_{2,4}$ and $c \neq 0$. So we have

$$
L^{5,3}+\Psi: \begin{cases}{\left[X_{0}, X_{i}\right]=X_{i+1}, 1 \leq i \leq 4,} & \left(Y_{1}, Y_{1}\right)=3 c X_{2} \\ {\left[X_{0}, Y_{i}\right]=Y_{i+1}, 1 \leq i \leq 2,} & \left(Y_{1}, Y_{2}\right)=\frac{3}{2} c X_{3} \\ {\left[X_{1}, Y_{1}\right]=a_{1} Y_{2},} & \left(Y_{1}, Y_{3}\right)=\frac{c}{2} X_{4} \\ {\left[X_{1}, Y_{2}\right]=\left(a_{1}-a_{2}\right) Y_{3},} & \left(Y_{2}, Y_{2}\right)=c X_{4} \\ {\left[X_{2}, Y_{1}\right]=a_{2} Y_{3},} & \left(Y_{2}, Y_{3}\right)=\frac{c}{2} X_{5}\end{cases}
$$

The condition $\Psi \circ \Psi\left(X_{1}, Y_{1}, Y_{2}\right)=0$ gives us $a_{1}=0$. Since $\Psi \circ \Psi\left(Y_{1}, Y_{1}, Y_{1}\right)=0$ we have $a_{2}=0$. With a change of scale we can consider $c=1$ and we obtain $L^{5,3}+\bar{\varphi}_{2,4}$.

- If $\mathfrak{g}_{\overline{0}}=Q_{5}$ we have to add the brackets $\left[X_{1}, X_{4}\right]=-X_{5}$ and $\left[X_{2}, X_{3}\right]=X_{5}$. Indeed, these brackets stem from cocycles of [26]. All the above reasoning is also valid, but from the condition $\Psi \circ \Psi\left(X_{1}, Y_{2}, Y_{2}\right)=0$ we obtain that $\left[X_{1}, X_{4}\right]=0$, which is clearly a contradiction. Thus, there is not any non-degenerated filiform Lie superalgebra deriving from the Lie algebra $Q_{5}$.

\section{$4.7 n=6$ and $m=3$}

Theorem 4.8 Let $\mathfrak{g}=\mathfrak{g}_{\overline{0}} \oplus \mathfrak{g}_{\overline{1}}$ be a non-degenerated filiform Lie superalgebra with $\operatorname{dim}\left(\mathfrak{g}_{\overline{0}}\right)=7$ and $\operatorname{dim}\left(\mathfrak{g}_{\overline{1}}\right)=3$. If $\mathfrak{g}$ is naturally graded, then the law of $\mathfrak{g}$ is isomorphic to the following law 


$$
L^{6,3}+\bar{\varphi}_{3,6}: \begin{cases}{\left[X_{0}, X_{i}\right]=X_{i+1}, 1 \leq i \leq 5,} & \left(Y_{1}, Y_{3}\right)=X_{4} \\ {\left[X_{0}, Y_{i}\right]=Y_{i+1}, 1 \leq i \leq 2,} & \left(Y_{2}, Y_{2}\right)=2 X_{4} \\ \left(Y_{1}, Y_{1}\right)=6 X_{2}, & \left(Y_{2}, Y_{3}\right)=X_{5} \\ \left(Y_{1}, Y_{2}\right)=3 X_{3}, & \left(Y_{3}, Y_{3}\right)=X_{6}\end{cases}
$$

Proof Let $\mathfrak{g}=\mathfrak{g}_{\overline{0}} \oplus \mathfrak{g}_{\overline{1}}$ be a naturally graded filiform Lie superalgebra with $\operatorname{dim}\left(\mathfrak{g}_{0}\right)=$ 5 and $\operatorname{dim}\left(\mathfrak{g}_{\overline{1}}\right)=3$. Therefore $\mathfrak{g}_{\overline{0}}=L_{6}$ and $\operatorname{gr}(\mathfrak{g})$ is

$$
\underbrace{<X_{0}, X_{1}, Y_{1}>}_{\mathfrak{g}^{1}} \oplus \underbrace{<X_{2}, Y_{2}>}_{\mathfrak{g}^{2}} \oplus \underbrace{<X_{3}, Y_{3}>}_{\mathfrak{g}^{3}} \oplus \underbrace{<X_{4}>}_{\mathfrak{g}^{4}} \oplus \underbrace{<X_{5}>}_{\mathfrak{g}^{5}} \oplus \underbrace{<X_{6}>}_{\mathfrak{g}^{6}}
$$

As in previous cases, since the products we get $L^{6,3}+\Psi$, with $\Psi=c \bar{\varphi}_{3,6}+a_{1} \Psi_{1,1}^{2}+$ $a_{2} \Psi_{2,1}^{3}$, where $\bar{\varphi}_{3,6}=6 \varphi_{1,2}+2 \varphi_{2,4}+\varphi_{3,6}$, and we have these products:

$$
L^{6,3}+\Psi: \begin{cases}{\left[X_{0}, X_{i}\right]=X_{i+1}, 1 \leq i \leq 5,} & \left(Y_{1}, Y_{2}\right)=3 c X_{3} \\ {\left[X_{0}, Y_{i}\right]=Y_{i+1}, 1 \leq i \leq 2,} & \left(Y_{1}, Y_{3}\right)=c X_{4} \\ {\left[X_{1}, Y_{1}\right]=a_{1} Y_{2},} & \left(Y_{2}, Y_{2}\right)=2 c X_{4} \\ {\left[X_{1}, Y_{2}\right]=\left(a_{1}-a_{2}\right) Y_{3},} & \left(Y_{2}, Y_{3}\right)=c X_{5} \\ {\left[X_{2}, Y_{1}\right]=a_{2} Y_{3},} & \left(Y_{3}, Y_{3}\right)=c X_{6} \\ \left(Y_{1}, Y_{1}\right)=6 c X_{2}, & \end{cases}
$$

with $c$ nonzero to avoid the trivial case. The condition $\Psi \circ \Psi\left(Y_{1}, Y_{1}, Y_{1}\right)=0$ gives us $a_{2}=0$, and since $\Psi \circ \Psi\left(X_{1}, Y_{1}, Y_{2}\right)=0$ we have $a_{1}=0$. By using a change of scale $c=1$ we obtain $L^{6,3}+\bar{\varphi}_{3,6}$.

\section{Naturally Graded (Non-Lie) Leibniz Superalgebras with Maximal Supernilindex}

Throughout this section we are going to study the Leibniz superalgebras with supernilindex $(n, m)$ because of the fact that in this case the even part $L_{\overline{0}}$ is a nullfiliform Leibniz algebra and the odd part $L_{\overline{1}}$ has structure of filiform $L_{\overline{0}}$-module. Consequently, it seems to be the first case to consider the "naturally graded" structure. Note that all these Leibniz superalgebras with supernilindex $(n, m)$ are non-Lie ones and contain in particular the only type of Leibniz superalgebra single generated or socalled null-filiform Leibniz superalgebras (for more details see [27]). It is not difficult to see that these last superalgebras, in the case of non-degenerated, are not naturally graded.

Next, we are going to recall the general expression of the Leibniz superalgebras with supernilindex $(n, m)$ (see [18]).

Theorem 5.1 If $L=L_{\overline{0}} \oplus L_{\overline{1}}$ is a Leibniz superalgebra of supernilindex $(n, m)$, then there exists an adapted basis of $L$, namely $\left\{X_{1}, X_{2}, \ldots, X_{n}, Y_{1}, Y_{2}, \ldots, Y_{m}\right\}$, with $\left\{X_{1}, X_{2}, \ldots, X_{n}\right\}$ a basis of $L_{\overline{0}}$ and $\left\{Y_{1}, Y_{2}, \ldots, Y_{m}\right\}$ a basis of $L_{\overline{1}}$, such that 


$$
\begin{aligned}
& {\left[X_{i}, X_{1}\right]=X_{i+1}, 1 \leq i \leq n-1,\left[X_{n}, X_{1}\right]=0} \\
& {\left[Y_{j}, X_{1}\right]=Y_{j+1}, 1 \leq j \leq m-1,\left[Y_{m}, X_{1}\right]=0}
\end{aligned}
$$

Moreover, $\left[Y_{j}, X_{k}\right]=0$ for $1 \leq j \leq m$ and $2 \leq k \leq n$, and the omitted products of $L_{\overline{0}}=<X_{1}, X_{2}, \ldots, X_{n}>$ vanish.

Thus, it can be seen that if $L=L_{\overline{0}} \oplus L_{\overline{1}}$ is a Leibniz superalgebra of snilindex $(n, m)$, then its graded superalgebra associated $\operatorname{gr}(L)=\sum_{i \in \mathbb{N}} L^{i}$, satisfying $\left[L^{i}, L^{j}\right] \subset L^{i+j}$, is exactly:

$$
\underbrace{<X_{1}, Y_{1}>}_{L^{1}} \oplus \underbrace{<X_{2}, Y_{2}>}_{L^{2}} \oplus \underbrace{<X_{3}, Y_{3}>}_{L^{3}} \oplus \cdots
$$

where for simplicity $L^{1}=L_{\overline{0}}^{1} \oplus L_{\overline{1}}^{1}=<X_{1}>\oplus<Y_{1}>$ has been replaced by $<X_{1}, Y_{1}>$ as there cannot be any possible confusion between even elements, called $X_{i}$, and odd ones, called $Y_{j}$. Analogously, the same replacement has been applied to every $L^{i}$. The last terms of $g r(L)$ depend on three possibilities:

1. If $n<m$, then

$$
\cdots \oplus \underbrace{<X_{n}, Y_{n}>}_{L^{n}} \oplus \underbrace{<Y_{n+1}>}_{L^{n+1}} \oplus \cdots \oplus \underbrace{<Y_{m}>}_{L^{m}}
$$

2. If $n=m$, then

$$
\cdots \oplus \underbrace{<X_{n-1}, Y_{n-1}>}_{L^{n-1}} \oplus \underbrace{<X_{n}, Y_{n}>}_{L^{n}}
$$

3. If $n>m$, then $\cdots \oplus \underbrace{<X_{m-1}, Y_{m}>}_{L^{m}} \oplus \underbrace{<X_{m}>}_{L^{m+1}} \oplus \cdots \oplus \underbrace{<X_{n}>}_{L^{n}}$

Remark 5.1 It can be seen that the even elements of $\operatorname{gr}(L)$, that is $\sum_{i \in \mathbb{N}} L_{\overline{0}}^{i}$, constitute a naturally graded null-filiform Leibniz algebra. That is,

$$
N F^{n}:\left[X_{i}, X_{1}\right]=X_{i+1}, \quad 1 \leq i \leq n-1,
$$

Furthermore, the definition of naturally graded Leibniz superalgebras is a generalization of naturally graded Leibniz algebras. Thus, if $L=L_{\overline{0}} \oplus L_{\overline{1}}$ is a naturally graded Leibniz superalgebra, then $L_{\overline{0}}$ is a naturally graded Leibniz algebra. Indeed, $L_{\overline{0}}^{i}$ is equivalent to $\mathcal{C}^{i-1}\left(L_{\overline{0}}\right) / \mathcal{C}^{i}\left(L_{\overline{0}}\right)$. Recall that the latter is used in the definition of naturally graded Leibniz algebras.

\subsection{Low Dimensions}

Next we are going to obtain all the naturally graded Leibniz superalgebras with supernilindex $(n, m)$ for low dimensions of either the even part or the odd part. We can accomplish such classification due to the results of [18]. 
Theorem 5.2 Let $L=L_{\overline{0}} \oplus L_{\overline{1}}$ be a non-degenerated naturally graded Leibniz superalgebra with s-nilindex $(n, m)$, then the law of $L$ is isomorphic to a law of the following list where the omitted products are equal to zero.

\section{List of laws.}

Pair of dimensions $n=2, m=2$. There is a one-parametric family of naturally graded Leibniz superalgebras of s-nilindex $(2,2)$ whose law can be expressed in an adapted basis $\left\{X_{1}, X_{2}, Y_{1}, Y_{2}\right\}$ as

$$
\mu_{1}^{\alpha}=\left\{\begin{array}{l}
{\left[X_{1}, X_{1}\right]=X_{2},\left[X_{1}, Y_{1}\right]=\alpha Y_{2}, \alpha \in \mathbb{C}} \\
{\left[Y_{1}, X_{1}\right]=Y_{2},\left[Y_{1}, Y_{1}\right]=X_{2}}
\end{array}\right.
$$

Pair of dimensions $n$ arbitrary with $n \geq 3, m=2$. The only sub-case in which there are naturally graded superalgebras is exactly $n=3$. In this case there exist two nonisomorphic naturally graded Leibniz superalgebras of s-nilindex $(3,2)$ whose laws can be expressed in an adapted basis $\left\{X_{1}, X_{2}, X_{3}, Y_{1}, Y_{2}\right\}$ by:

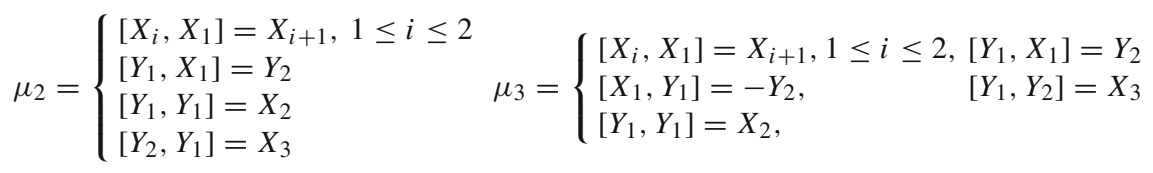

Pair of dimensions $n=2, m=3$. There are two non-isomorphic naturally graded Leibniz superalgebras of s-nilindex $(2,3)$ whose laws can be expressed in an adapted basis $\left\{X_{1}, X_{2}, Y_{1}, Y_{2}, Y_{3}\right\}$ by:

$$
\mu_{1}=\left\{\begin{array}{l}
{\left[X_{1}, X_{1}\right]=X_{2},} \\
{\left[Y_{1}, X_{1}\right]=Y_{2}} \\
{\left[Y_{2}, X_{1}\right]=Y_{3}} \\
{\left[Y_{1}, Y_{1}\right]=X_{2}}
\end{array} \mu_{3}=\left\{\begin{array}{l}
{\left[X_{1}, X_{1}\right]=X_{2},\left[Y_{1}, X_{1}\right]=Y_{2}} \\
{\left[X_{1}, Y_{1}\right]=-Y_{2},\left[Y_{2}, X_{1}\right]=Y_{3}} \\
{\left[X_{1}, Y_{2}\right]=-Y_{3},\left[Y_{1}, Y_{1}\right]=X_{2}}
\end{array}\right.\right.
$$

Pair of dimensions $n=3, m=3$. There are two non-isomorphic naturally graded Leibniz superalgebras of s-nilindex $(3,3)$ whose laws can be expressed in an adapted basis $\left\{X_{1}, X_{2}, X_{3}, Y_{1}, Y_{2}, Y_{3}\right\}$ by:

$$
\mu_{1}=\left\{\begin{array}{l}
{\left[X_{i}, X_{1}\right]=X_{i+1}, 1 \leq i \leq 2} \\
{\left[Y_{j}, X_{1}\right]=Y_{j+1}, 1 \leq j \leq 2} \\
{\left[Y_{1}, Y_{2}\right]=X_{3}} \\
{\left[Y_{2}, Y_{1}\right]=-X_{3}}
\end{array} \mu_{8}=\left\{\begin{array}{l}
{\left[X_{i}, X_{1}\right]=X_{i+1}, 1 \leq i \leq 2} \\
{\left[Y_{j}, X_{1}\right]=Y_{j+1}, 1 \leq j \leq 2} \\
{\left[X_{1}, Y_{2}\right]=-Y_{3}} \\
{\left[Y_{1}, Y_{1}\right]=X_{2}} \\
{\left[Y_{1}, Y_{2}\right]=X_{3}}
\end{array}\right.\right.
$$

Pair of dimensions $n=4, m=3$. There are two non-isomorphic naturally graded Leibniz superalgebras of s-nilindex $(4,3)$ whose laws can be expressed in an adapted basis $\left\{X_{1}, X_{2}, X_{3}, X_{4}, Y_{1}, Y_{2}, Y_{3}\right\}$ by: 


$$
\mu_{9}=\left\{\begin{array}{l}
{\left[X_{i}, X_{1}\right]=X_{i+1}, 1 \leq i \leq 3,\left[Y_{1}, Y_{3}\right]=X_{4}} \\
{\left[Y_{j}, X_{1}\right]=Y_{j+1}, 1 \leq j \leq 2,\left[Y_{2}, Y_{2}\right]=-X_{4}} \\
{\left[X_{1}, Y_{1}\right]=-Y_{2},} \\
{\left[X_{1}, Y_{2}\right]=-Y_{3}} \\
{\left[Y_{3}, Y_{1}\right]=X_{4}}
\end{array} \mu_{12}=\left\{\begin{array}{l}
{\left[X_{i}, X_{1}\right]=X_{i+1}, 1 \leq i \leq 3} \\
{\left[Y_{j}, X_{1}\right]=Y_{j+1}, 1 \leq j \leq 2} \\
{\left[Y_{1}, Y_{1}\right]=X_{2}} \\
{\left[Y_{2}, Y_{1}\right]=X_{3}} \\
{\left[Y_{3}, Y_{1}\right]=X_{4}}
\end{array}\right.\right.
$$

Pair of dimensions $n$ arbitrary with $n \geq 5, m=3$. There is not any naturally graded Leibniz superalgebra of s-nilindex $(n, 3)$.

Pair of dimensions $n=2$, $m$ arbitrary with $m \geq 4$. There are two non-isomorphic naturally graded Leibniz superalgebras of s-nilindex $(2, m)$ whose laws can be expressed in an adapted basis $\left\{X_{1}, X_{2}, Y_{1}, Y_{2}, Y_{3}, Y_{4}, \cdots, Y_{m}\right\}$ by

$$
\mu_{m-1}=\left\{\begin{array}{l}
{\left[X_{1}, X_{1}\right]=X_{2}} \\
{\left[Y_{i}, X_{1}\right]=Y_{i+1}, 1 \leq i \leq m-1} \\
{\left[Y_{1}, Y_{1}\right]=X_{2}}
\end{array} \mu_{m+1}=\left\{\begin{array}{l}
{\left[X_{1}, X_{1}\right]=X_{2}} \\
{\left[X_{1}, Y_{j}\right]=-Y_{j+1}, 2 \leq j \leq m-1} \\
{\left[Y_{i}, X_{1}\right]=Y_{i+1}, \quad 1 \leq i \leq m-1} \\
{\left[Y_{1}, Y_{1}\right]=X_{2}}
\end{array}\right.\right.
$$

\subsection{Cases $n \geq 3$ and $m \geq 4$}

Throughout this sub-section we deal with the problem of determining the structure of the aforementioned naturally graded Leibniz superalgebras for the remaining dimensions. Notice that the naturally graded Leibniz superalgebra with maximal s-nilindex named $N G^{n, m}$ appears in every pair of arbitrary dimensions $n$ and $m$.

$$
N G^{n, m}:\left\{\begin{array}{l}
{\left[X_{i}, X_{1}\right]=X_{i+1}, 1 \leq i \leq n-1} \\
{\left[Y_{j}, X_{1}\right]=Y_{j+1}, 1 \leq j \leq m-1} \\
{\left[Y_{i}, Y_{1}\right]=X_{i+1}, 1 \leq i \leq \min \{n-1, m\}}
\end{array}\right.
$$

with the vectors $\left\{X_{1}, X_{2}, \ldots, X_{n}, Y_{1}, Y_{2}, \ldots, Y_{m}\right\}$ as adapted basis. Also, we have the following result.

Theorem 5.3 Let $L=L_{\overline{0}} \oplus L_{\overline{1}}$ be a non-degenerated naturally graded Leibniz superalgebras with s-nilindex $(n, m)$ and adapted basis $\left\{X_{1}, X_{2}, \ldots, X_{n}, Y_{1}, Y_{2}, \ldots, Y_{m}\right\}$. Then the law of $L$ will be isomorphic either to the law named $N G^{n, m}$ or to a law belonging to the following families of laws. The precise expression of these last families of laws depends on two possibilities, that is:

1. If $n \leq m$,

$$
\begin{cases}{\left[X_{i}, X_{1}\right]=X_{i+1},} & 1 \leq i \leq n-1, \\
{\left[Y_{j}, X_{1}\right]=Y_{j+1},} & 1 \leq j \leq m-1, \\
{\left[X_{1}, Y_{j}\right]=-Y_{j+1},} & 1 \leq j \leq m-1, \\
{\left[Y_{1}, Y_{1}\right]=\gamma_{1} X_{2},} & 3 \leq i \leq n-1, \\
{\left[Y_{i}, Y_{1}\right]=\gamma_{i} X_{i+1},} & \\
{\left[Y_{i}, Y_{j}\right]=\sum_{s=0}^{j-1}(-1)^{s}\left(\begin{array}{c}
j-1 \\
s
\end{array}\right) \gamma_{i+s} X_{i+j},} & 1 \leq i \leq n-2,2 \leq j \leq n-i-1 .\end{cases}
$$


with $\gamma_{2}=0$ and $\gamma_{2 j}=(-1)^{j} \sum_{s=0}^{j-2}(-1)^{s}\left(\begin{array}{c}j-1 \\ s\end{array}\right) \gamma_{j+s+1}$, for $2 \leq j \leq\left\lfloor\frac{n-1}{2}\right\rfloor$.

2. If $n>m$,

$$
\begin{cases}{\left[X_{i}, X_{1}\right]=X_{i+1},} & 1 \leq i \leq n-1, \\
{\left[Y_{j}, X_{1}\right]=Y_{j+1},} & 1 \leq j \leq m-1, \\
{\left[X_{1}, Y_{j}\right]=-Y_{j+1},} & 1 \leq j \leq m-1, \\
{\left[Y_{1}, Y_{1}\right]=\gamma_{1} X_{2},} & 3 \leq i \leq m, \\
{\left[Y_{i}, Y_{1}\right]=\gamma_{i} X_{i+1},} & \\
{\left[Y_{i}, Y_{j}\right]=\sum_{s=0}^{\min \{j-1, m-i\}}(-1)^{s}\left(\begin{array}{c}
j-1 \\
s
\end{array}\right) \gamma_{i+s} X_{i+j},} & 2 \leq j \leq m, 1 \leq i \leq \min \{m, n-j\} .\end{cases}
$$

with $\gamma_{2}=0$ and

$$
\gamma_{2 j}=(-1)^{j} \sum_{s=0}^{j-2}(-1)^{s}\left(\begin{array}{c}
j-1 \\
s
\end{array}\right) \gamma_{j+s+1}, 2 \leq j \leq\left\lfloor\frac{m}{2}\right\rfloor \quad \gamma_{i}=\sum_{s=1}^{m-i}(-1)^{s+1}\left(\begin{array}{c}
m \\
s
\end{array}\right) \gamma_{i+s} .
$$

\section{\lfloor\rfloor denotes the whole part of the number.}

Proof An adequate use of Mathematica software has been truly helpful to accomplish the proof of this theorem. It has been a very useful tool to both complete all the calculations involved in many particular pair of dimensions and deduce the process to follow in arbitrary dimensions. Next, we will distinguish two cases. We only prove case 1 in details and omit case 2 completely in order not to repeat the same ideas.

Case 1: $n \leq m$. We consider the adapted basis $\left\{X_{1}, X_{2}, \ldots, X_{n}, Y_{1}, Y_{2}, \ldots, Y_{m}\right\}$ and the gradation

$<X_{1}, Y_{1}>\oplus<X_{2}, Y_{2}>\oplus \cdots<X_{n}, Y_{n}>\oplus<Y_{n+1}>\oplus<Y_{n+2}>\oplus \cdots \oplus<Y_{m}>$

Using their properties

$$
\begin{array}{ll}
{\left[X_{i}, X_{1}\right]=X_{i+1}, \quad 1 \leq i \leq n-1,} & {\left[Y_{j}, X_{1}\right]=Y_{j+1}, \quad 1 \leq j \leq m-1,} \\
{\left[X_{1}, Y_{j}\right]=\alpha_{j} Y_{j+1}, 1 \leq j \leq m-1,} & {\left[X_{i}, Y_{j}\right]=\beta_{i j} Y_{i+j}, 2 \leq i \leq n, \quad 1 \leq j \leq m-i} \\
{\left[Y_{i}, Y_{j}\right]=\gamma_{i j} X_{i+j}, 1 \leq i \leq m, \quad 1 \leq j \leq n-i .} &
\end{array}
$$

We have that $\left[X_{1}, Y_{1}\right]+\left[Y_{1}, X_{1}\right] \in \operatorname{Ann}(L)$, then $\left(\alpha_{1}+1\right) Y_{2} \in \operatorname{Ann}(L)$.

Case 1.1. $1+\alpha_{1} \neq 0$, then $Y_{2} \in A n n(L)$. Taking into account that $\left[Y_{2}, X_{1}\right]=Y_{3}$, it is easy to prove that $Y_{3} \in \operatorname{Ann}(L)$. Analogously, $Y_{4}, Y_{5}, \ldots, Y_{m} \in \operatorname{Ann}(L)$. Thus, we have

$$
\begin{aligned}
& {\left[X_{i}, X_{1}\right]=X_{i+1}, \quad 1 \leq i \leq n-1,\left[Y_{j}, X_{1}\right]=Y_{j+1}, \quad 1 \leq j \leq m-1,} \\
& {\left[X_{1}, Y_{1}\right]=\beta_{1} Y_{2}, \quad\left[X_{i}, Y_{1}\right]=\beta_{i} Y_{i+1}, 2 \leq i \leq n,} \\
& {\left[Y_{i}, Y_{1}\right]=\gamma_{i} X_{i+1}, 1 \leq i \leq n-1 .}
\end{aligned}
$$

We make the super Leibniz identity on the triples $\left\{Y_{i}, Y_{1}, X_{1}\right\}$, and we obtain $\gamma_{i}=\gamma_{1}=\gamma$ with $1 \leq i \leq n-1$. If $\gamma=0$, then the superalgebra is degenerated; thus, $\gamma \neq 0$ and making $Y_{1}^{\prime}=\frac{1}{\sqrt{\gamma}} Y_{1}$ allows us to consider $\gamma=1$. 
Now, from the super Leibniz identity on the triples $\left\{X_{i}, Y_{1}, Y_{1}\right\}$ for $1 \leq i \leq n-2$ we get $\beta_{i}=0$. From $\left\{X_{n-2}, Y_{1}, X_{1}\right\}$ and $\left\{X_{n-1}, Y_{1}, X_{1}\right\}$ we obtain $\beta_{n-1}=0$ and $\beta_{n}=0$, respectively.

Thus, we obtain the naturally graded Leibniz superalgebra $N G^{n, m}$.

Case 1.2. $1+\alpha_{1}=0$. We can distinguish two cases:

(a) $1+\alpha_{i}=0$ for $2 \leq i \leq m-1$. We have:

$$
\begin{aligned}
& {\left[X_{i}, X_{1}\right]=X_{i+1}, \quad 1 \leq i \leq n-1, \quad\left[Y_{j}, X_{1}\right]=Y_{j+1}, \quad 1 \leq j \leq m-1,} \\
& {\left[X_{1}, Y_{j}\right]=-Y_{j+1}, \quad 1 \leq j \leq m-1, \quad\left[X_{i}, Y_{j}\right]=\beta_{i j} Y_{i+j}, 2 \leq i \leq n, \quad 1 \leq j \leq m-i} \\
& {\left[Y_{i}, Y_{j}\right]=\gamma_{i j} X_{i+j}, 1 \leq i \leq m, \quad 1 \leq j \leq n-i \text {. }}
\end{aligned}
$$

The super Leibniz identity on the following triples imposes further constraints on the above family.

\begin{tabular}{lll}
\hline Super Leibniz identity & Constraint \\
\hline$\left\{X_{1}, X_{1}, Y_{j},\right\}, 1 \leq j \leq m-2$ & $\Rightarrow$ & $\beta_{2 j}=0$ \\
$\left\{X_{1}, X_{i}, Y_{j}\right\}, 3 \leq i \leq n, 1 \leq j \leq m-i-1$ & $\Rightarrow$ & $\beta_{i j}=0$ and \\
& $\Rightarrow$ & {$\left[X_{i}, Y_{m-i}\right]=\beta_{i m-i} Y_{m}=\beta_{i} Y_{m}, 3 \leq i \leq n$} \\
$\left\{X_{i}, X_{1}, Y_{m-i-1},\right\}, 2 \leq i \leq n-1$ & $\beta_{3}=0$ and $\beta_{i+1}=(-1)^{i+1} \beta_{3}=0$ \\
$\left\{Y_{i}, X_{1}, Y_{j-1}\right\}, 1 \leq i \leq n-2,2 \leq j \leq n-i$ & $\Rightarrow$ & $\gamma_{i j}=\gamma_{i j-1}-\gamma_{i+1 j-1}$ \\
\hline
\end{tabular}

Thus, we prove by the induction method that:

$\gamma_{i 1}=\gamma_{i}, 1 \leq i \leq n-1, \quad \gamma_{i j}=\sum_{k=0}^{j-1}(-1)^{k}\left(\begin{array}{c}j-1 \\ k\end{array}\right) \gamma_{i+k}, \quad 1 \leq i \leq n-2,2 \leq j \leq n-i$

Applying the above relations, we can write

$$
\begin{array}{ll}
{\left[X_{i}, X_{1}\right]=X_{i+1},} & 1 \leq i \leq n-1, \quad\left[Y_{j}, X_{1}\right]=Y_{j+1}, \quad 1 \leq j \leq m-1 \\
{\left[X_{1}, Y_{j}\right]=-Y_{j+1},} & 1 \leq j \leq m-1,\left[Y_{i}, Y_{1}\right]=\gamma_{i} X_{i+1}, 1 \leq i \leq n-1 \\
{\left[Y_{i}, Y_{j}\right]=\sum_{k=0}^{j-1}(-1)^{k}\left(\begin{array}{c}
j-1 \\
k
\end{array}\right) \gamma_{i+k} X_{i+j}, 1 \leq i \leq n-2, \quad 2 \leq j \leq n-i}
\end{array}
$$

For instance, the super Leibniz identity on the triples $\left[X_{1},\left[Y_{1}, Y_{1}\right]\right]$ and $\left[X_{1},\left[Y_{j}, Y_{j}\right]\right]$ gives, respectively, $\gamma_{2}=0$ and

$$
\gamma_{2 j}=(-1)^{j} \sum_{s=0}^{j-2}(-1)^{s}\left(\begin{array}{c}
j-1 \\
s
\end{array}\right) \gamma_{j+s+1}, 2 \leq j \leq\left\lfloor\frac{n-1}{2}\right\rfloor .
$$

We obtain the family of naturally graded Leibniz superalgebras with maximal snilindex of the theorem statement.

(b) There exists $k$ such that $1+\alpha_{i}=0$ for $2 \leq i \leq k-2$, and $1+\alpha_{k-1} \neq 0$. We have

$$
\left[X_{1}, Y_{k-1}\right]+\left[Y_{k-1}, X_{1}\right]=\left(1+\alpha_{k-1}\right) Y_{k} \in \operatorname{Ann}(L) .
$$


Then $Y_{k} \in A n n(L)$ and $Y_{k+1}, Y_{k+2}, \ldots, Y_{m} \in A n n(L)$. Hence, we can consider

$$
\begin{aligned}
& {\left[X_{i}, X_{1}\right]=X_{i+1}, \quad 1 \leq i \leq n-1, \quad\left[Y_{j}, X_{1}\right]=Y_{j+1}, \quad 1 \leq j \leq m-1,} \\
& {\left[X_{1}, Y_{j}\right]=-Y_{j+1}, \quad 1 \leq j \leq k-2, \quad\left[X_{1}, Y_{k-1}\right]=\alpha Y_{k}, 1+\alpha \neq 0} \\
& {\left[X_{i}, Y_{j}\right]=\beta_{i j} Y_{i+j}, 1 \leq j \leq k-1, \quad 2 \leq i \leq \min \{n, m-j\}} \\
& {\left[Y_{i}, Y_{j}\right]=\gamma_{i j} X_{i+j}, 1 \leq j \leq k-1, \quad 1 \leq i \leq n-k+1 \text {. }}
\end{aligned}
$$

Consider the super Leibniz identity on the triple $\left\{Y_{i}, X_{1}, Y_{j}\right\}$ with $2 \leq j \leq k-1$ and $1 \leq i \leq n-j$. We get

$$
\gamma_{i j}=\gamma_{i j-1}-\gamma_{i+1} j-1 \text {. }
$$

For $j=k$, we have that $\gamma_{i-1}=\gamma_{1 k-1}$. If we rename $\gamma_{i 1}:=\gamma_{i}$ with $1 \leq i \leq n-1$, it is easy to prove by induction method on $j$, that

$$
\gamma_{i j}=\sum_{s=0}^{j-1}(-1)^{s}\left(\begin{array}{c}
j-1 \\
s
\end{array}\right) \gamma_{i+s}, \quad 2 \leq j \leq k-1, \quad 1 \leq i \leq n-j
$$

Thus, we have that

$$
\begin{array}{ll}
{\left[Y_{i}, Y_{1}\right]=\gamma_{i} X_{i+1},} & 1 \leq i \leq n-1 \\
{\left[Y_{i}, Y_{j}\right]=\sum_{s=0}^{j-1}(-1)^{s}\left(\begin{array}{c}
j-1 \\
s
\end{array}\right) \gamma_{i+s} X_{i+j}, 2 \leq j \leq k-1, \quad 1 \leq i \leq n-j}
\end{array}
$$

From the super Leibniz identity on the triple $\left\{X_{1}, Y_{1}, Y_{1}\right\}$ we get $\gamma_{2}=0$.

Using the induction method together with super Leibniz identity on the triple $\left\{X_{i}, X_{1}, Y_{j}\right\}$, we prove that

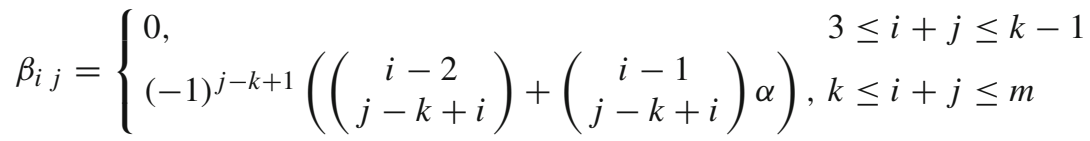

with $2 \leq i \leq \min \{n, m-j\}$.

(1) $\mathbf{m} \geq \mathbf{n}+\mathbf{3}$. If we make the super Leibniz identity on the triples $\left\{X_{n}, X_{1}, Y_{1}\right\}$ and $\left\{X_{n}, X_{1}, Y_{2}\right\}$ we get $\alpha=-\frac{k-2}{n}$ and $\alpha=-\frac{k-3}{n}$, respectively. But that is a contradiction, then there is not any superalgebra.

(2) $\mathbf{m}=\mathbf{n}+\mathbf{2}$.

On the other hand, recall that $\gamma_{j}=0$, for $k-2 \leq j \leq n-1$, and by Equation (5.1) we have that $\gamma_{k j}=0$. Accordingly $\gamma_{j+1 k-1}=0$ for $1 \leq j \leq n-k$, that is,

$$
\sum_{s=0}^{k-2}(-1)^{s}\left(\begin{array}{c}
k-2 \\
s
\end{array}\right) \gamma_{j+1+s}=0, \quad 1 \leq j \leq n-k
$$




\begin{tabular}{lll}
\hline Super Leibniz identity & & Constraint \\
$\left\{X_{n}, X_{1}, Y_{1},\right\}$ & $\Rightarrow$ & $\alpha=-\frac{k-2}{n}$. \\
$\left\{Y_{i}, Y_{1}, Y_{n+1-i}\right\}$ & $\Rightarrow$ & $\left\{\begin{array}{l}\gamma_{i} \beta_{i+1 n+1-i}=0, \\
\beta_{i+1 n+1-i \neq 0,} \neq 0, \quad \Rightarrow \gamma_{i}=0, n+2-k \leq i \leq n-1 . \\
i \geq n+2-k \\
\gamma_{i} \beta_{i+1}=0, \\
\beta_{i+11} \neq 0, \\
k-2 \leq i \leq n\end{array} \Rightarrow \gamma_{i}=0, k-2 \leq i \leq n-1\right.$. \\
$\left\{Y_{i}, Y_{1}, Y_{1}\right\}$ & $\Rightarrow$ \\
$\left\{X_{1}, Y_{k-1}, Y_{j}\right\}$ & $\Rightarrow$
\end{tabular}

Then we have a system of $(n-k)$ linear equations and $(n-k)$ variables admitting one unique solution $\gamma_{i}=0,2 \leq i \leq n-k+1$.

Only rest to prove that $\gamma_{1}=0$. For this, it is sufficient to consider the super Leibniz identity on the triple $\left\{Y_{1}, Y_{1}, Y_{k-1}\right\}$. We conclude that the superalgebra is degenerated. (3) $\mathbf{m}=\mathbf{n}+\mathbf{1}$. From the super Leibniz identity on $\left\{Y_{i}, Y_{1}, Y_{1}\right\}$ we get $\gamma_{i} \beta_{i+1}=0$. If $k-2 \leq i \leq n$ then $\beta_{i+1} \neq 0$. Thus $\gamma_{i}=0$ for $k-2 \leq i \leq n-1$.

Analogously, from $\left\{Y_{k-3}, Y_{1}, Y_{3}\right\},\left\{X_{1}, Y_{1}, Y_{j}\right\}$ we conclude that $\gamma_{k-3}=0$, and $\gamma_{2 j}+\gamma_{j+1}=0$, respectively. On the other hand, remember that $\gamma_{j}=0$ with $k-2 \leq$ $j \leq n-1$, which implies that

$$
\sum_{s=1}^{j-2}(-1)^{s}\left(\begin{array}{c}
j-1 \\
s
\end{array}\right) \gamma_{2+s}+\left((-1)^{j-1}+1\right) \gamma_{j+1}=0, \quad 4 \leq j \leq k-3
$$

Then we have a system of $(k-6)$ linear equations and $(k-6)$ variables admitting one unique solution $\gamma_{i}=0$ with $3 \leq i \leq k-4$.

Only rest to prove that $\gamma_{1}=0$. For this, it is sufficient to consider the super Leibniz identity on the triples $\left\{Y_{1}, Y_{1}, Y_{k-1}\right\}$ if $k$ is odd and $\left\{Y_{1}, Y_{1}, Y_{k-2}\right\}$ if $k$ is even. We conclude then that the superalgebra is degenerated.

Case 2. $n>m$. We consider the adapted basis $\left\{X_{1}, X_{2}, \ldots, X_{n}, Y_{1}, Y_{2}, \ldots, Y_{m}\right\}$ and the gradation

$<X_{1}, Y_{1}>\oplus<X_{2}, Y_{2}>\oplus \cdots<X_{m}, Y_{m}>\oplus<X_{m+1}>\oplus<X_{m+2}>\oplus \cdots \oplus<X_{n}>$

By using the adapted basis and the properties of natural gradation

$$
\begin{aligned}
& {\left[X_{i}, X_{1}\right]=X_{i+1}, \quad 1 \leq i \leq n-1, \quad\left[Y_{j}, X_{1}\right]=Y_{j+1}, \quad 1 \leq j \leq m-1} \\
& {\left[X_{1}, Y_{j}\right]=\alpha_{j} Y_{j+1}, \quad 1 \leq j \leq m-1,\left[X_{i}, Y_{j}\right]=\beta_{i j} Y_{i+j}, \quad 1 \leq j \leq m-2, \quad 2 \leq i \leq m-j} \\
& {\left[Y_{i}, Y_{j}\right]=\gamma_{i j} X_{i+j}, 1 \leq i \leq m, \quad 1 \leq j \leq \min \{n-i, m\}}
\end{aligned}
$$

Case 2.1. If $1+\alpha_{1} \neq 0$, then $Y_{2}, Y_{3}, \ldots, Y_{m} \in A n n(L)$. We have

$$
\begin{array}{ll}
{\left[X_{i}, X_{1}\right]=X_{i+1},} & 1 \leq i \leq n-1,\left[Y_{j}, X_{1}\right]=Y_{j+1}, \quad 1 \leq j \leq m-1 \\
{\left[X_{1}, Y_{1}\right]=\alpha Y_{2},} & {\left[X_{i}, Y_{1}\right]=\beta_{i} Y_{i+1}, 2 \leq i \leq m-1} \\
{\left[Y_{i}, Y_{1}\right]=\gamma_{i} X_{i+1}, 1 \leq i \leq m} &
\end{array}
$$


Similar to the previous cases, using the super Leibniz identity, we get the superalgebra $N G^{n, m}$.

Case 2.2. $1+\alpha_{1}=0$. We distinguish the following two cases:

(a) $1+\alpha_{i}=0$ for all $i, 2 \leq i \leq m-1$. We have:

$$
\begin{aligned}
& {\left[X_{i}, X_{1}\right]=X_{i+1}, \quad 1 \leq i \leq n-1, \quad\left[Y_{j}, X_{1}\right]=Y_{j+1}, \quad 1 \leq j \leq m-1} \\
& {\left[X_{1}, Y_{j}\right]=-Y_{j+1}, \quad 1 \leq j \leq m-1,\left[X_{i}, Y_{j}\right]=\beta_{i j} Y_{i+j}, \quad 1 \leq j \leq m-2, \quad 2 \leq i \leq m} \\
& {\left[Y_{i}, Y_{j}\right]=\gamma_{i} X_{i+j}, 1 \leq i \leq m, \quad 1 \leq j \leq \min \{m, n-i\}}
\end{aligned}
$$

We obtain the family of naturally graded Leibniz superalgebras with maximal snilindex of the theorem statement.

(b) There exists $k$, with $2 \leq k \leq m-1$, such that $1+\alpha_{i}=0$ for $2 \leq i \leq k-2$ and $1+\alpha_{k-1} \neq 0$. We have

$$
\left[X_{1}, Y_{k-1}\right]+\left[Y_{k-1}, X_{1}\right]=\left(1+\alpha_{k-1}\right) Y_{k} \in \operatorname{Ann}(L) .
$$

Then $Y_{k} \in A n n(L)$ and $Y_{k+1}, Y_{k+2}, \ldots, Y_{m} \in A n n(L)$. Thus,

$$
\begin{aligned}
& {\left[X_{i}, X_{1}\right]=X_{i+1}, \quad 1 \leq i \leq n-1,} \\
& {\left[X_{1}, Y_{j}\right]=-Y_{j+1}, \quad 1 \leq j \leq k-2,} \\
& {\left[X_{i}, Y_{j}\right]=\beta_{i j} Y_{i+j}, 1 \leq j \leq k-1, \quad 2 \leq i \leq m-j} \\
& {\left[Y_{i}, Y_{j}\right]=\gamma_{i j} X_{i+j}, 1 \leq j \leq k-1, \quad 1 \leq i \leq \min \{m, n-j\}} \\
& {\left[Y_{j}, X_{1}\right]=Y_{j+1}, \quad 1 \leq j \leq m-1} \\
& {\left[X_{1}, Y_{k-1}\right]=\alpha Y_{k}, 1+\alpha \neq 0}
\end{aligned}
$$

By using the same techniques as in the case 1 , we conclude that the obtained superalgebra is degenerated.

\section{References}

1. Adashev, J.K., Camacho, L.M., Omirov, B.A.: Central extensions of null-filiform and naturally graded filiform non-Lie Leibniz algebras. J. Algebra 479, 461-486 (2017)

2. Albeverio, S., Ayupov, ShA, Omirov, B.A.: On nilpotent and simple Leibniz algebras. Commun. Algebra 33(1), 159-172 (2005)

3. Albeverio, S., Omirov, B.A., Khudoyberdiyev, A.K.: On the classification of complex Leibniz superalgebras with characteristic sequence $\left(n-1,1 \mid m_{1}, \ldots, m_{k}\right)$ and nilindex $n+m$. J. Algebra Appl. 8(4), 461-475 (2009)

4. Albeverio, S., Omirov, B.A., Rakhimov, I.S.: Varieties of nilpotent complex Leibniz algebras of dimension less than five. Commun. Algebra 33(5), 1575-1585 (2005)

5. Ancochea Bermúdez, J.M., Campoamor-Stursberg, R., García Vergnolle, L.: Classification of Lie algebras with naturally graded quasi-filiform nilradicals. J. Geom. Phys. 61(11), 2168-2186 (2011)

6. Ayupov, ShA, Omirov, B.A.: On some classes of nilpotent Leibniz algebras. Sib. Math. J. 42(1), 15-24 (2001)

7. Ayupov, ShA, Omirov, B.A.: On Leibniz Algebras. Algebra and Operator Theory (Tashkent 1997), pp. 1-12. Kluwer Academic Publishers, Dordrecht (1998)

8. Bordemann, M., Gómez, J.R., Khakimdjanov, Y., Navarro, R.M.: Some deformations of nilpotent Lie superalgebras. J. Geom. Phys. 57(5), 1391-1403 (2007)

9. Burde, D., Steinhoff, C.: Classification of orbit closures of 4-dimensional complex Lie algebras. J. Algebra 214, 729-739 (1999)

10. Cabezas, J.M., Pastor, E.: Naturally graded $p$-filiform Lie algebras in arbitrary finite dimension. J. Lie Theory 15(2), 379-391 (2005)

11. Camacho, L.M., Gómez, J.R., Omirov, B.A., Khudoyberdiyev, A.K.: On description of Leibniz superalgebras of nilindex $n+m$. Forum Math. 24(4), 809-826 (2012) 
12. Camacho, L.M., Gómez, J.R., Omirov, B.A., Khudoyberdiyev, A.K.: Complex nilpotent Leibniz superalgebras with nilindex equal to dimension. Commun. Algebra 41(7), 2720-2735 (2013)

13. Casas, J.M., Ladra, M., Omirov, B.A., Karimjanov, I.A.: Classification of solvable Leibniz algebras with naturally graded filiform nilradical. Linear Algebra Appl. 438(7), 2973-3000 (2013)

14. Fuks, D.B.: Cohomology of Infinite-dimensional Lie Algebras. Contemporary Soviet Mathematics. Consultants Bureau, New York (1986)

15. Gómez, J.R., Jiménez-Merchán, A.: Naturally graded quasi-filiform Lie algebras. J. Algebra 256(1), 211-228 (2002)

16. Gómez, J.R., Khakimdjanov, Yu., Navarro, R.M.: Some problems concerning to nilpotent Lie superalgebras. J. Geom. Phys. 51(4), 473-486 (2004)

17. Gómez, J.R., Khakimdjanov, Yu., Navarro, R.M.: Infinitesimal deformations of the Lie superalgebra $L^{n, m}$. J. Geom. Phys. 58(7), 849-859 (2008)

18. Gómez, J.R., Omirov, B.A.: Navarro R.M. On nilpotent Leibniz superalgebras. arXiv:math/0611723

19. Gómez, J.R., Omirov, B.A., Khudoyberdiyev, A.K.: The classification of Leibniz superalgebras of nilindex $n+m(m \neq 0)$. J. Algebra 324(10), 2786-2803 (2010)

20. Goze, M., Khakimdjanov, Y.: Nilpotent Lie Algebras. Mathematics and its Applications, Kluwer Academic Publishers Group, Dordrecht (1996). ISBN 0-7923-3932-0

21. Grunewald, F., O'Halloran, J.: Varieties of nilpotent Lie algebras of dimension less than six. J. Algebra 112(2), 315-326 (1988)

22. Jacobson, N.: Lie Algebras. Tracts in Pure and Applied Mathematics. Interscience Publishers (a division of John Wiley \& Sons), New York (1962)

23. Khakimdjanov, Y.: Varieties of Lie algebra laws. Handb. Algebr., pp. 509-541. Elsevier, Amsterdam (2000)

24. Khakimdjanov, Y.: Affine structures on filiform Lie algebras. Lie groups and invariant theory, pp. 141-155, American. Math. Soc. Transl. Ser. 2, 213, Adv. Math. Sci. vol. 56, American Mathematical Society, Providence (2005)

25. Khakimdjanov, Yu., Navarro, R.M.: A complete description of all the infinitesimal deformations of the Lie superalgebra $L^{n, m}$. J. Geom. Phys. 60(1), 131-141 (2010)

26. Khakimdjanov, Y., Navarro, R.M.: Deformations of filiform Lie algebras and superalgebras. J. Geom. Phys. 60(9), 1156-1169 (2010)

27. Khudoyberdiyev, A.K., Omirov, B.A.: Infinitesimal deformations of null-filiform Leibniz superalgebras. J. Geom. Phys. 74, 370-380 (2013)

28. Ladra, M., Masutova, K.K., Omirov, B.A.: Corrigendum to "Classification of solvable Leibniz algebras with naturally graded filiform nilradical” [Linear Algebra Appl. 438 (7) (2013) pp. 2973-3000]. Linear Algebra Appl. 507 (2016), pp. 513-517

29. Livernet, M.: Rational homotopy of Leibniz algebras. Manuscripta Math. 96(3), 295-315 (1998)

30. Loday, J.-L.: Une version non commutative des algèbres de Lie: les algèbres de Leibniz. (French). Enseign. Math. (2) 39(3-4), 269-293 (1993)

31. Navarro, R.M.: Low-dimensional filiform Lie superalgebras. J. Geom. Phys. 108, 71-82 (2016)

32. Scheunert, M.: The Theory of Lie Superalgebras. An Introduction. Lecture Notes in Mathematics. Springer, Berlin (1979). ISBN: 3-540-09256-0

33. Scheunert, M., Zhang, R.B.: Cohomology of Lie superalgebras and of their generalizations. J. Math. Phys. 39(9), 5024-5061 (1998)

34. Vergne, M.: Cohomologie des algèbres de Lie nilpotentes. application à l'étude de la variété des algèbres de Lie nilpotentes. (French). Bull. Soc. Math. France 98, 81-116 (1970) 